A computational study of two-phase viscoelastic systems in a capillary tube with a sudden contraction/expansion

Daulet Izbassarov and Metin Muradoglu

Citation: Phys. Fluids 28, 012110 (2016); doi: 10.1063/1.4939940

View online: http://dx.doi.org/10.1063/1.4939940

View Table of Contents: http://aip.scitation.org/toc/phf/28/1

Published by the American Institute of Physics

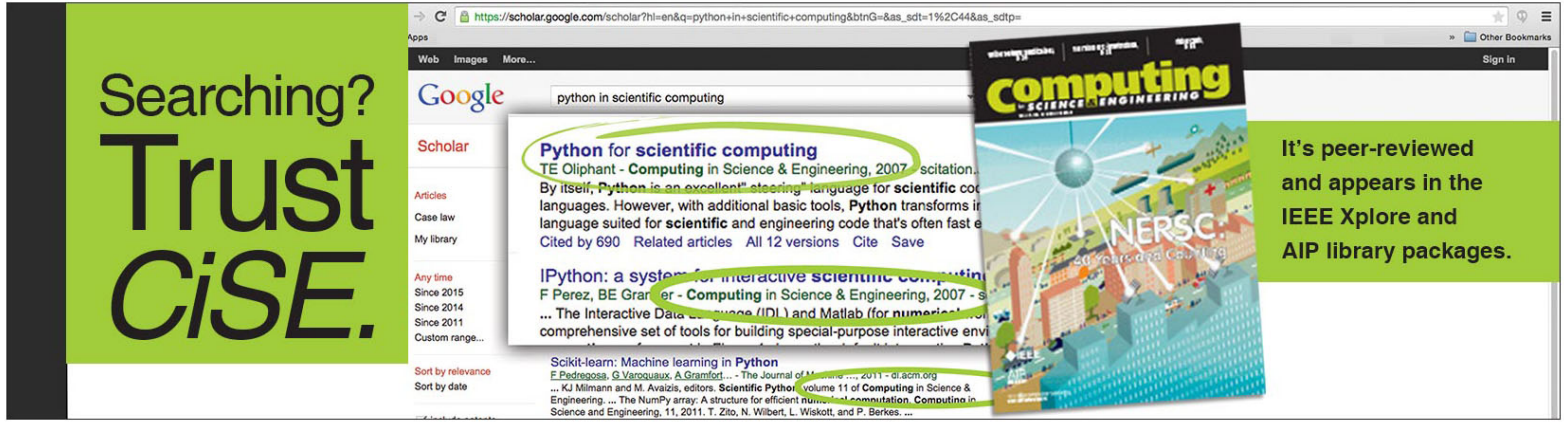




\title{
A computational study of two-phase viscoelastic systems in a capillary tube with a sudden contraction/expansion
}

\author{
Daulet Izbassarov and Metin Muradoglua) \\ Department of Mechanical Engineering, Koç University, Rumelifeneri Yolu, \\ 34450 Sariyer, Istanbul, Turkey
}

(Received 12 June 2015; accepted 28 December 2015; published online 26 January 2016)

\begin{abstract}
Two-phase viscoelastic systems are computationally studied in a pressure-driven flow with a sudden contraction and expansion using a finite-difference/front-tracking method. The effects of viscoelasticity in drop and bulk fluids are investigated including high Weissenberg and Reynolds number cases up to $W i=100$ and $R e=100$. The Finitely Extensible Non-linear Elastic-Chilcott and Rallison (FENE-CR) model is used to account for the fluid viscoelasticity. Extensive computations are performed to examine drop dynamics for a wide range of parameters. It is found that viscoelasticity interacts with drop interface in a non-monotonic and complicated way, and the two-phase viscoelastic systems exhibit very rich dynamics especially in the expansion region. At high $R e$, the drop undergoes large deformation in the contraction region followed by strong shape oscillations in the downstream of the expansion. For a highly viscous drop, a re-entrant cavity develops in the contraction region at the trailing edge which, in certain cases, grows and eventually causes encapsulation of ambient fluid. The re-entrant cavity formation is initiated at the entrance of the contraction and is highly influenced by the viscoelasticity. Compared to the corresponding straight channel case, the effects of viscoelasticity are reversed in the constricted channel: Viscoelasticity in drop/continuous phase hinders/enhances formation of the re-entrant cavity and entrainment of ambient fluid into main drop. Encapsulation of ambient fluid into main droplet may be another route to produce a compound droplet in microfluidic applications. @ 2016 AIP Publishing LLC. [http://dx.doi.org/10.1063/1.4939940]
\end{abstract}

\section{INTRODUCTION}

Confined multiphase fluid dynamics is of fundamental importance in a wide range of engineering applications and natural processes such as the droplet-based microfluidics, ${ }^{1,2}$ enhanced oil recovery, ${ }^{3}$ blood cells in microcirculation, ${ }^{4-7}$ polymer blends, and polymer processing. ${ }^{8}$ Viscoelastic effects play a significant role in all these applications often in the presence of confinement. Viscoelastic liquids exhibit a range of exotic behaviors that can be utilized to perform useful functions especially in microfluidic applications. For instance, microfluidic memory and control device, ${ }^{9}$ microfluidic rectifier, ${ }^{10}$ nonlinear viscoelastic flow resistor, ${ }^{9}$ synthesis of non-spherical particles,${ }^{11}$ and enhanced mixing in microchannels ${ }^{12}$ rely on viscoelasticity of working fluids. With the rapidly growing popularity of droplet-based microfluidic devices, there has been significant interest recently in viscoelastic two-phase systems in confined geometries. ${ }^{13-16}$ It is thus crucially important to understand viscoelastic effects on drop dynamics in confined geometries for development of various lab-on-a-chip devices.

Unlike viscous systems, understanding of viscoelasticity is severely limited, and yet of great importance for flow modeling and simulation. The difficulty is due to complex rheological behavior of the fluids which often leads to controversies. ${ }^{17-21}$ For instance, there were contradictory observations about the effects of viscoelasticity on drop deformation, i.e., whether viscoelasticity increases or decreases drop deformation in a shear flow. ${ }^{17,18}$ Numerical simulations of Yue et al. ${ }^{21}$ and Aggarwal

\footnotetext{
a) Author to whom correspondence should be addressed. Electronic mail: mmuradoglu@ku.edu.tr
} 
and Sarkar ${ }^{22}$ recently resolved this issue and found a non-monotonic dependence of deformation on viscoelasticity with an initially decreasing followed by an increasing trend. In a uniform extensional flow, reduced deformation is predicted for a viscoelastic drop in a viscous medium and the enhanced deformation otherwise. ${ }^{23,24}$ This is consistent with the heuristic idea that viscoelasticity in the drop phase opposes deformation, while in the matrix facilitates it. Also there were contradictory results about the effects of fluid elasticity on drop deformation in a converging conical channel. ${ }^{19,25,26}$ Experimental works of Chin and $\mathrm{Han}^{25}$ and Mighri et al. ${ }^{19}$ demonstrated similar droplet deformation and dynamics in this geometry as are for a uniform extensional flow. On the contrary, the numerical study of Khayat ${ }^{26}$ showed that viscoelastic effects in the drop assist deformation while in the matrix impede it. A more recent computational study of Zhou $\mathrm{et} \mathrm{al.}{ }^{27}$ demonstrated a more complex picture in which viscoelasticity in either phases may suppress or promote drop deformation depending on the capillary number and the drop-to-matrix viscosity ratio.

Confined multiphase flows have recently received a special attention due to the growing interest in microfluidic technologies. ${ }^{4,28,29}$ There have been a few experimental studies ${ }^{30-32}$ on confined motion of viscoelastic two-phase systems, e.g., motion of neutrally/non-neutrally buoyant drops suspended in a Newtonian/viscoelastic Stokes flow. Ho and Leal ${ }^{30}$ and Olbricht and Leal ${ }^{32}$ demonstrated that viscoelasticity of the suspending fluid significantly influences the droplet dynamics in straight and constricted capillary tubes. Recently, Khobdeh ${ }^{31}$ studied deformation and breakup of viscoelastic two-phase systems in capillaries of different cross sections. For cylindrical and rectangular channels, an increase in elasticity of drop phase inhibits deformation, while in suspending fluid, it has non-monotonic effect: a decrease followed by an increase. Khobdeh ${ }^{31}$ also investigated drop dynamics in periodically constricted capillary tubes and found that drop deformation is enhanced by viscoelasticity irrespective of which phase is elastic. $\mathrm{Wu}^{33}$ numerically investigated both buoyancy-driven and pressure-driven motions of viscoelastic drop in a capillary tube. He found that elasticity in the drop phase suppresses drop deformation. You et al. ${ }^{34}$ conducted numerical simulations for a confined buoyancy-driven drop in various viscoelastic two-phase systems. They concluded that viscoelasticity in the interior phase pulls the trailing end inward, while in the exterior fluid, pulls it out.

Unlike single phase case, viscoelastic multiphase flows through a contraction/expansion geometry have received less attention. When a drop passes through a contraction it experiences regions of strong shearing near the walls: non-homogeneous uniaxial extension along the centerline upstream of the contraction plane and non-homogeneous biaxial expansion downstream of the expansion. Extensive simulations have been done by Harvie and co-workers ${ }^{35-37}$ to understand a Newtonian and a generalized non-Newtonian drop dynamics in an axisymmetric 4:1:4 contraction/expansion geometry. Viscoelastic effects on drop deformation have been also investigated by several other groups in axisymmetric ${ }^{38}$ and planar ${ }^{15,39,40}$ configurations for similar geometries. Zhou et al. ${ }^{38}$ simulated the deformation of an axisymmetric viscoelastic/Newtonian droplet to model fluid dynamics of a neutrophil moving through a constriction in a Newtonian environment. However, their simulations were restricted to low Reynolds number (creeping) flows with viscoelasticity contained only in the drop phase and a capillary tube without any expansion region. Harvie et al. ${ }^{15}$ considered a viscoelastic drop moving in a Newtonian medium in a planar channel with a sudden contraction/expansion. They demonstrated qualitative agreement between computational results and experimental observations. In particular, they observed the forked tail formation in the contraction region. Chung et al. ${ }^{40}$ investigated two-phase systems in a planar channel with a 5:1:5 constriction/expansion region and considered cases in which either droplet or ambient fluid is viscoelastic. They found that viscoelasticity significantly enhances drop deformation especially at high viscosity ratios for a Newtonian drop in a viscoelastic medium while it has much less effects on drop deformation for the case of a viscoelastic droplet in a Newtonian fluid. Note that they considered relatively low Weissenberg number cases, i.e., up to $W i=O(1)$.

The inertial effects on drop deformation have been often neglected in previous studies. However, in spite of the common wisdom that inertia is negligible in microfluidic systems, recent studies have shown that a variety of inertial effects can be utilized for various applications in microchannels including enhanced mixing, particle separation, and bioparticle focusing ${ }^{41,42}$ Amini et al.${ }^{41}$ reviewed a variety of mechanisms by which inertia and asymmetry in channel or particle geometry can be used to control particles and flows in microfluidic platforms. Moreover, inertia can significantly promote 
the deformation of microdroplets for the Reynolds number in the range of $R e=1-100.43,44$ To our knowledge, there has been no numerical study to investigate the effects of viscoelasticity and inertia on drop dynamics in an axisymmetric tube with a sudden contraction/expansion.

In the present study, extensive direct numerical simulations are carried out to examine the effects of viscoelasticity on drop dynamics in various two-phase systems in a pressure-driven tube with a 4:1:4 sudden contraction/expansion. The inertial effects are fully taken into account by solving the Navier-Stokes and viscoelastic model equations in the entire computational domain using the front-tracking method. ${ }^{45,46}$ We consider the cases of a Newtonian droplet in a viscoelastic medium (NV), a viscoelastic droplet in a Newtonian medium (VN) and a viscoelastic droplet in another viscoelastic medium (VV). The fluid viscoelasticity is accounted for using the Finitely Extensible Non-linear Elastic-Chilcott and Rallison (FENE-CR) model. ${ }^{47}$ Simulations are performed to investigate drop deformation and dynamics for the $\mathrm{NV}, \mathrm{VN}$, and VV systems in a contraction/expansion geometry for a wide range of parameters including the relative droplet size $(\kappa)$, the capillary number $(\mathrm{Ca})$, Reynolds number $(\mathrm{Re})$, viscosity ratio $(\theta)$, and the viscoelasticity characterized by the Weissenberg number $(W i)$, solvent viscosity ratio $(\beta)$, and the extensibility parameter $\left(L^{2}\right)$. In addition, the effects of the inner and outer Weissenberg numbers $\left(W i_{i}\right.$ and $\left.W i_{o}\right)$ as well as their ratio $(k)$ are also examined for the VV system.

The main contributions of the present work can be summarised as follows:

1. This is the first direct numerical simulation study of pressure-driven viscoelastic two-phase flow systems in an axisymmetric channel with a sudden contraction and expansion at finite Reynolds and Weissenberg numbers. The previous computational studies were restricted to planar twodimensional geometries, ${ }^{15,39,40}$ the only exception being the numerical study by Zhou et al ${ }^{38}$ who considered an axisymmetric channel with only a smooth constriction but without an expansion region.

2. Combined inertial and viscoelastic effects on drop dynamics are studied for the Weissenberg and Reynolds numbers up to $W i=100$ and $R e=100$. The previous computational studies were limited to low Reynolds number flows $\left(R e=O(1)\right.$ or smaller) in the VN system ${ }^{15,38}$ or to low Weissenberg numbers (i.e., $W i=O(1)){ }^{39,40}$ It is found that a drop undergoes a large deformation in the narrow pipe followed by strong shape oscillations in the expansion region at high $R e$ numbers and these oscillations interact strongly with the viscoelasticity in a non-monotonic and complicated way.

3. Combined viscous and viscoelastic effects on drop dynamics are also investigated for the NV and VN systems. It is found that a highly viscous drop may develop a re-entrant cavity at the trailing edge which eventually leads to encapsulation of ambient fluid into main droplet in some cases. This phenomenon is highly influenced by viscoelasticity non-monotonically in a complicated and somewhat unexpected way. Compared to the corresponding straight channel case, the effects of viscoelasticity are reversed in the constricted channel: Viscoelasticity in drop/continuous phase hinders/enhances formation of the re-entrant cavity and entrainment of ambient fluid into main drop. This result may indicate that the viscoelasticity can be used to create a compound droplet with a precise volume of the inner and outer droplets.

4. The FENE-CR model employed in the present study is more realistic than the commonly used Oldroyd-B model since it accounts for the finite polymer extensibility. Thus, the present results are expected to better represent the physics of two-phase viscoelastic fluid systems.

The rest of this paper is organized as follows: In Sec. II, we briefly describe the governing equations including the constitutive model for viscoelasticity and numerical method. The physical problem and flow geometry are described in Sec. III. The results are presented and discussed in Sec. IV. Conclusions are drawn in Sec. V. An extensive grid convergence study is also presented in the Appendix.

\section{FORMULATION AND NUMERICAL METHOD}

The governing equations are described in the framework of the finite difference/front tracking method. The flow is assumed to be incompressible. Following the work of Unverdi and Tryggva$\operatorname{son}^{46}$ and Izbassarov and Muradoglu, ${ }^{45}$ a single set of governing equations can be written for the 
entire computational domain provided that the jumps in the material properties such as density, viscosity and relaxation time are taken into account and the effects of the interfacial surface tension are treated appropriately.

The continuity and momentum equations can be written as follows:

$$
\begin{aligned}
\nabla \cdot \mathbf{u} & =0, \\
\frac{\partial \rho \mathbf{u}}{\partial t}+\nabla \cdot(\rho \mathbf{u u}) & =-\nabla p+\nabla \cdot \mu_{s}\left(\nabla \mathbf{u}+\nabla \mathbf{u}^{T}\right)+\nabla \cdot \tau+\int_{A} \sigma \kappa \mathbf{n} \delta\left(\mathbf{x}-\mathbf{x}_{\mathbf{f}}\right) d A,
\end{aligned}
$$

where $\mu_{s}, \rho, p, \mathbf{u}$, and $\boldsymbol{\tau}$ denote the solvent viscosity and the density of the fluid, the pressure, the velocity vector, and the viscoelastic extra stress tensor, respectively. The last term in Eq. (2) represents the body force due to surface tension where $\sigma$ is the surface tension coefficient, $\kappa$ is twice the mean curvature, and $\mathbf{n}$ is the unit vector normal to the interface. The surface tension acts only on the interface as indicated by the three-dimensional delta function, $\delta$, whose arguments $\mathbf{x}$ and $\mathbf{x}_{\mathbf{f}}$ are the points at which the equation is being evaluated and a point at the interface, respectively.

The FENE-CR model is adopted as the constitutive equation for the viscoelastic extra stresses. This model can be written as

$$
\begin{aligned}
\frac{\partial \mathbf{A}}{\partial t}+\nabla \cdot(\mathbf{u A})-(\nabla \mathbf{u})^{T} \cdot \mathbf{A}-\mathbf{A} \cdot \nabla \mathbf{u} & =-\frac{F_{A}}{\lambda}(\mathbf{A}-\mathbf{I}), \\
F_{A} & =\frac{L^{2}}{L^{2}-\operatorname{trace}(\mathbf{A})},
\end{aligned}
$$

where $\mathbf{A}, \lambda, L, F_{A}$, and $\mathbf{I}$ are the conformation tensor, the relaxation time, the extensibility parameter defined as the ratio of the length of a fully extended polymer dumbbell to its equilibrium length, the stretch function, and the identity tensor, respectively. The extra stress tensor $\tau$ is related to the conformation tensor as

$$
\tau=\frac{F_{A} \mu_{p}}{\lambda}(\mathbf{A}-\mathbf{I})
$$

where $\mu_{p}$ is the polymeric viscosity.

It is also assumed that the material properties remain constant following a fluid particle, i.e.,

$$
\frac{D \rho}{D t}=0, \frac{D \mu_{s}}{D t}=0, \frac{D \mu_{p}}{D t}=0, \frac{D \lambda}{D t}=0,
$$

where $\frac{D}{D t}=\frac{\partial}{\partial t}+\mathbf{u} \cdot \nabla$ is the material derivative. The density, polymeric and solvent viscosities, and the relaxation time vary discontinuously across the fluid interface are given by

$$
\begin{aligned}
\mu_{p} & =\mu_{p, i} \phi+\mu_{p, o}(1-\phi), \mu_{s}=\mu_{s, i} \phi+\mu_{s, o}(1-\phi), \\
\rho & =\rho_{i} \phi+\rho_{o}(1-\phi), \lambda=\lambda_{i} \phi+\lambda_{o}(1-\phi),
\end{aligned}
$$

where the subscripts $i$ and $o$ denote the properties of the drop and the bulk fluids, respectively. The indicator function $\phi$ is defined such that it is unity inside the droplet and zero outside.

The flow equations (Eqs. (1) and (2)) are solved fully coupled with the viscoelastic model equations (Eq. (3)) by the finite-difference/front-tracking method developed by Izbassarov and $\mathrm{Mu}-$ radoglu. ${ }^{45}$ The flow and viscoelastic model equations are solved on a staggered Eulerian grid using a projection method. ${ }^{48}$ The log-conformation method (LCM) of Fattal and Kupferman ${ }^{49}$ is employed to enhance numerical stability in solving the viscoelastic model equations at high Weissenberg numbers. A fifth-order upwinded Weighted Essentially Non-Oscillatory (WENO-Z) scheme of Borges et al. ${ }^{50}$ is used to discretize the convective terms in the viscoelastic constitutive equations while all the other spatial derivatives are approximated using central differences. Time integration is achieved using a first order explicit Euler method both for the flow and viscoelastic model equations. Although it is straightforward to make the present numerical method second order accurate in time, a first order method is used here since the time stepping error is generally found to be negligibly small compared to the spatial error mainly due to a small time step imposed by the numerical stability of the present explicit scheme. ${ }^{45}$

A separate Lagrangian grid is used to explicitly track the fluid-fluid interface. The Lagrangian grid consists of linked marker points (the front) moving with the local flow velocity interpolated from 
the stationary Eulerian grid. The piece of the Lagrangian grid between two marker points is called a front element. The surface tension is computed on the Lagrangian grid and then distributed onto the Eulerian grid points near the interface to be added to the momentum equations in the form of body forces as described by Tryggvason et al. ${ }^{51}$ The indicator function is computed at each time step based on the location of the interface using the standard procedure ${ }^{51}$ and is then used to set the fluid properties in each phase. The Lagrangian grid is restructured at every time step by deleting the front elements that are smaller than a prespecified lower limit and by splitting the front elements that are larger than a prespecified upper limit in the same way as described by Tryggvason et al. ${ }^{51}$ to keep the front element size nearly uniform and comparable to the Eulerian grid size. It is critically important to restructure the Lagrangian grid since it avoids unresolved wiggles due to small elements and lack of resolution due to large elements. A complete description of the front-tracking method can be found in the work of Tryggvason et al. ${ }^{51}$ and the treatment of the viscoelasticity in the work of Izbassarov and Muradoglu. ${ }^{45}$

\section{PROBLEM STATEMENT}

The physical problem and computational domain are sketched in Fig. 1. The flow is assumed to be axisymmetric so only one half is used as the computational domain. The capillary tube contains a 4:1:4 contraction/expansion section. The radii of the main channel and the contraction are $R_{1}$ and $R_{2}$, respectively. The total length of the tube is varied depending on the Reynolds number $(R e)$. At higher $R e$, due to oscillation of the drop in the expansion region, longer tube is required to reach a steady-state. The tube has a total length of $60 R_{2}$ and $130 R_{2}$ for the range of $2 \leq R e<10$ and $10 \leq R e \leq 100$, respectively. A sudden constriction of length $20 R_{2}$ is located at $20 R_{2}$ from the upstream as shown in Fig. 1. A spherical droplet of diameter $d_{d}$ is placed instantly in a distance of $10 R_{2}$ from the inlet. The flow is initiated instantaneously by imposing a fully developed steady flow at the inlet and keeping the pressure constant at the outlet. Symmetry and no-slip boundary conditions are applied at the centerline and at the wall of the tube, respectively. The viscoelastic stress tensor at the inlet is specified based on the analytical solution assuming a fully developed pipe flow. The Neumann boundary conditions are used for all viscoelastic stress components at the other boundaries. The governing equations are solved in their dimensional forms but the results are expressed in terms of relevant non-dimensional quantities. Using the average flow velocity $V$ and the channel radius $R_{2}$ in the narrow part of channel as the velocity and length scales, respectively, and the time scale $R_{2} / V$,
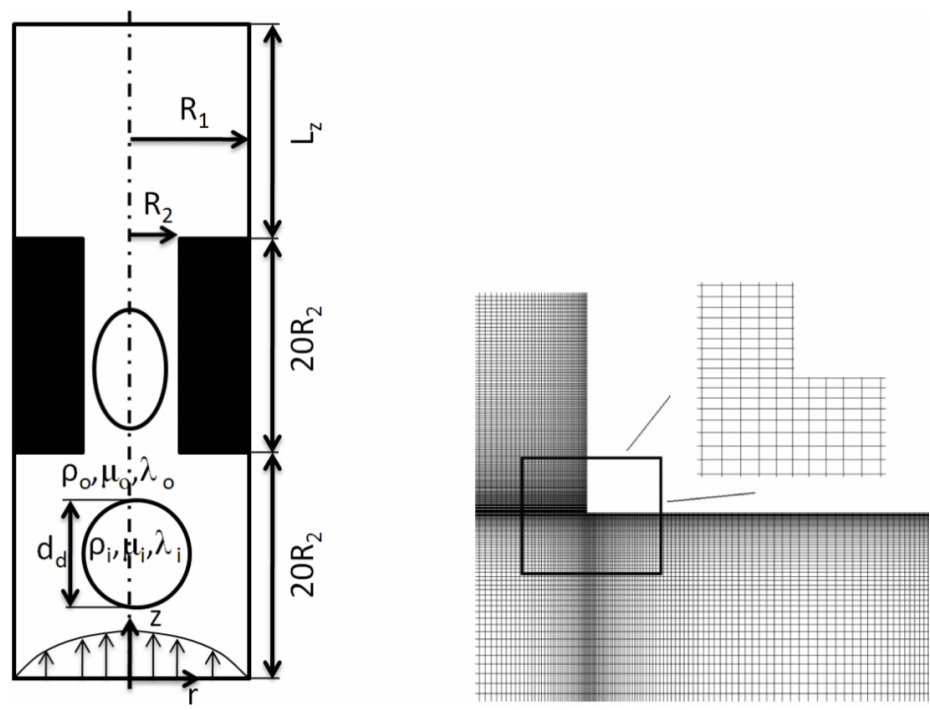

FIG. 1. (left) Schematic illustration of a pressure-driven viscoelastic two phase system. (right) Mesh near the re-entrant corner. 
the governing non-dimensional numbers are defined as

$$
C a=\frac{\mu_{o} V}{\sigma}, R e=\frac{\rho_{o} V R_{2}}{\mu_{o}}, W i=\frac{\lambda V}{R_{2}}, \theta=\frac{\mu_{i}}{\mu_{o}}, \alpha=\frac{\rho_{i}}{\rho_{o}}, k=\frac{\lambda_{i}}{\lambda_{o}}, \beta=\frac{\mu_{s}}{\mu_{p}+\mu_{s}}, \kappa=\frac{d_{d}}{2 R_{2}},
$$

where $\mathrm{Ca}, \mathrm{Re}$, and $\mathrm{Wi}$ are the capillary, Reynolds, and Weissenberg numbers, respectively. The other parameters $\theta, \alpha, k$, and $\beta$ denote the total viscosity, density, relaxation time, and solvent viscosity ratios, respectively. The relative droplet size $\kappa$ is used as a measure for the confinement. The drop deformation is defined as

$$
\text { deformation }=\frac{d_{a}-d_{r}}{d_{a}+d_{r}},
$$

where $d_{a}$ and $d_{r}$ are the maximum droplet dimensions in the axial and radial directions, respectively. The density ratio $\alpha$ is fixed to be unity, i.e., $\rho_{i}=\rho_{o}$, in all the results presented in this paper. Simulations are performed by varying only one parameter while keeping all the others constant in order to demonstrate the sole effects of the parameter on the flow. To facilitate this, we define a base case as $\theta=1, \kappa=1.25, \operatorname{Re}=2, C a=0.1, W i=1, L^{2}=25$, and $\beta=0.5$. Note that the base case is selected to be consistent with the range of parameters used by Chung et al. ${ }^{39,40}$

\section{RESULTS AND DISCUSSIONS}

Extensive simulations are carried out to study the dynamics of viscoelastic two-phase systems in a pressure driven axisymmetric contraction/expansion capillary tube. A non-uniform Cartesian grid (Fig. 1) is employed in the computations. The grid is stretched near the contraction and expansion regions, where the largest stress gradients occur. A comprehensive grid convergence study is performed to determine the minimum grid size required to reduce the spatial discretization error below a threshold value. As detailed in the Appendix, for $R e<10$, a grid with 64 cells in the radial direction is sufficient to reduce the numerical error below $4 \%$ for all the flow quantities and thus this grid is used in all relevant computations presented in this paper. A finer grid containing 96 cells in the radial direction is used for $R e>10$ and found to reduce the spatial error below $2 \%$ for all the flow quantities except for the case of the VN system at very high Weissenberg numbers (i.e., Wi $=100$ ) for which the maximum error becomes as large as $9 \%$ in the vicinity of the expansion. Note that, although not shown here, the drop volume is preserved within $2 \%$ for all the cases considered in this study.

Simulations are first performed to examine the effects of various flow parameters on the drop dynamics in the NV, VN, and VV systems at a low Reynolds number, i.e., $R e=2$. Note that the Reynolds number is set to $R e=O(1)$ to relax the restriction on time step due to numerical stability and thus to facilitate extensive numerical simulations. Then further simulations are performed to demonstrate the combined effects of inertia and viscoelasticity for the Reynolds and Weissenberg numbers up to $R e=100$ and $W i=100$.

\section{A. Computations at a low Reynolds number $(R e=2)$}

\section{Effects of confinement $(\mathrm{K})$}

First the effects of confinement on the drop dynamics are examined in the constriction region. The confinement is characterized by the relative droplet size $\kappa$ and it is varied between $0.5 \leq \kappa \leq 1.75$ while keeping all other parameters fixed at their values in the base case. The results are shown in Fig. 2 for the VN case. The square root of the trace of conformation tensor $(\sqrt{\operatorname{trace}(\mathbf{A})})$ is plotted as a measure of average polymer length. The enlarged version of the droplet in the middle of the constriction is also illustrated on the right side of each case in Fig. 2 to better show the details of drop deformation and viscoelastic stress distribution. Similar to the all Newtonian case, ${ }^{52}$ the drop shape is asymmetric, i.e., the curvature at the fore is higher than that at the aft, due to the pressure gradient in the axial direction. Large viscoelastic stresses are generated at the sides of the drop due to high shear stresses exerted by the continuous fluid, and then these stresses are convected by the internal circulation to create a stress concentration near the rear stagnation point, as shown in Fig. 2. Similar 


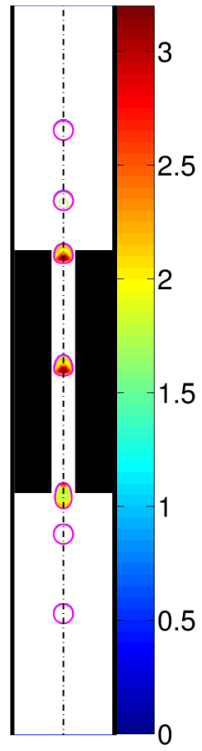

$\kappa=0.8$

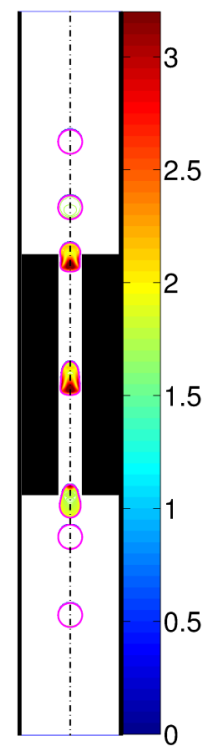

$\kappa=1$

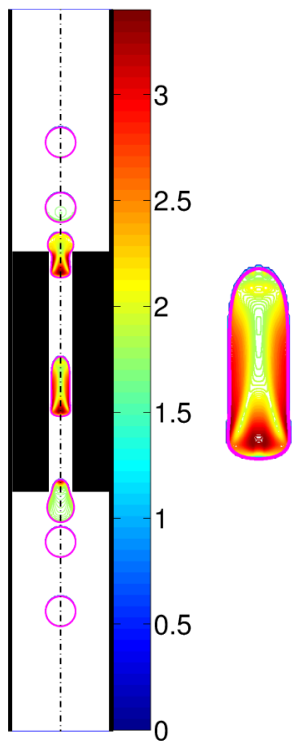

$\kappa=1.25$

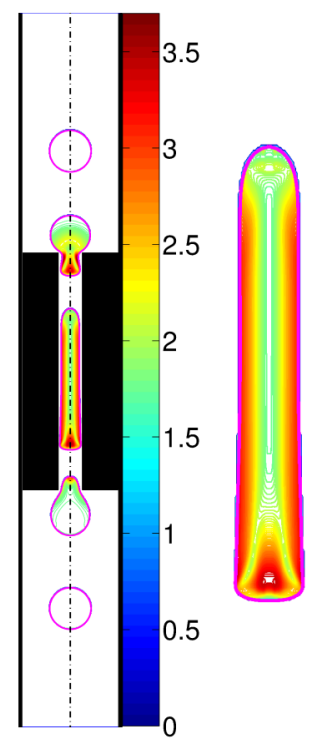

$\kappa=1.75$

FIG. 2. The effects of the relative drop size $(\kappa)$ for the VN case. The evolution of droplet shape and contours of average polymer extension $\sqrt{\operatorname{trace}(\mathbf{A})}$ are plotted for a FENE-CR droplet moving in a Newtonian fluid. The enlarged version of the drop in the middle of the constriction is also shown on the right side of each case $(\mathrm{Ca}=0.1, R e=2, \mathrm{Wi}=1$, grid: $64 \times 768)$.

to the planar case studied by Chung et al., ${ }^{39}$ the droplet takes a bullet like shape at low confinement and becomes more elongated as $\kappa$ increases. The drop deformation is plotted in Fig. 3 against the dimensionless moving distance of the drop centroid defined as $z_{d} / 2 R_{2}$ where $z_{d}$ is the axial distance of the drop centroid from its initial location. Note that the narrow pipe is located between $z_{d} / 2 R_{2}=5$ and $z_{d} / 2 R_{2}=15$. The drop undergoes a significant deformation for $\kappa \geq 0.8$ and the deformation grows rapidly as $\kappa$ is further increased. In the entrance region, there is an overshoot in the drop deformation, which is more pronounced for smaller droplets. This overshoot is caused by the retraction of droplet after it enters the constriction. All the droplets reach a steady shape except for the largest one (i.e., $\kappa=1.75)$ in the narrow channel. In the expansion region, all drops swell towards the cross-stream direction making the deformation parameter negative and eventually reach a nearly spherical shape in the further downstream.

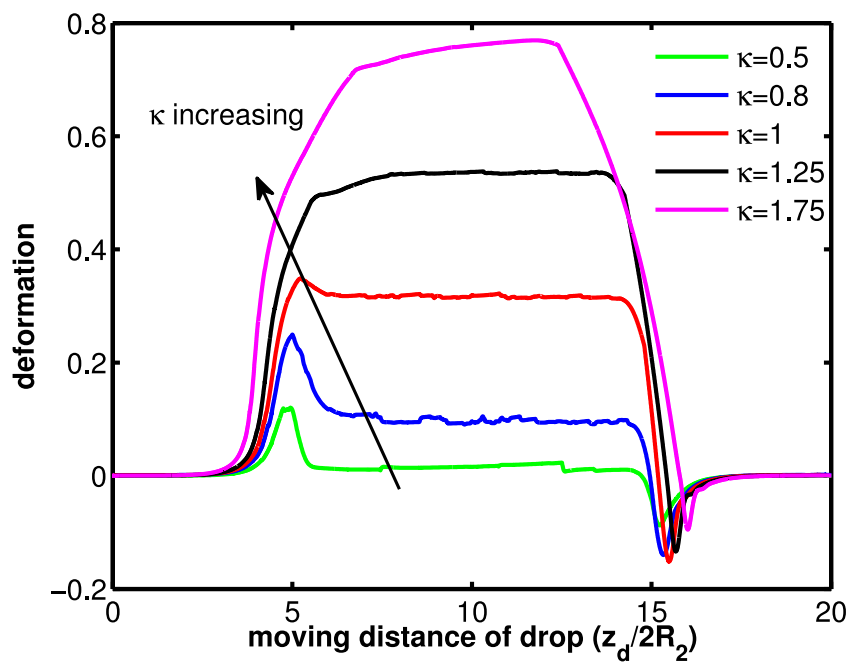

FIG. 3. The effects of the relative drop size $(\kappa)$ for the VN case. Drop deformation is plotted against the moving distance of the drop centroid for various $\kappa$ ranging between $\kappa=0.5$ and $\kappa=1.75(C a=0.1, R e=2, W i=1$, grid: $64 \times 768)$. 


\section{Effects of Weissenberg number (Wi)}

Next, we investigate the viscoelastic effects on drop dynamics at $R e=2$. For low $R e$, viscoelastic effects are especially pronounced in the constriction region. It is known that the drop shape is strongly influenced by viscoelastic normal stress difference $A_{z z}-A_{r r}{ }^{39,40,45}$ The drop shapes together with the contours of constant normal stress differences are shown in Figs. 4 and 5 for the VN and NV cases, respectively, for various combinations of $\mathrm{Ca}$ and $\mathrm{Wi}$ numbers. Since a steady droplet shape is not attained by the end of the constriction for high $\mathrm{Ca}$ and $\mathrm{Wi}$ number cases, the results are shown when the droplets are approximately at the middle of the constriction. For the VN case (Fig. 4), as $W i$ increases, the viscoelastic stresses increase in magnitude and penetrate further into the bulk of the drop. It is worth noting that, in contrast with the Oldroyd-B model, the FENE-CR model bounds the growth of normal stress difference. Therefore the increase in the maximum normal stress difference gets smaller when Wi exceeds a threshold value as can be seen in Fig. 4. Furthermore, decreasing
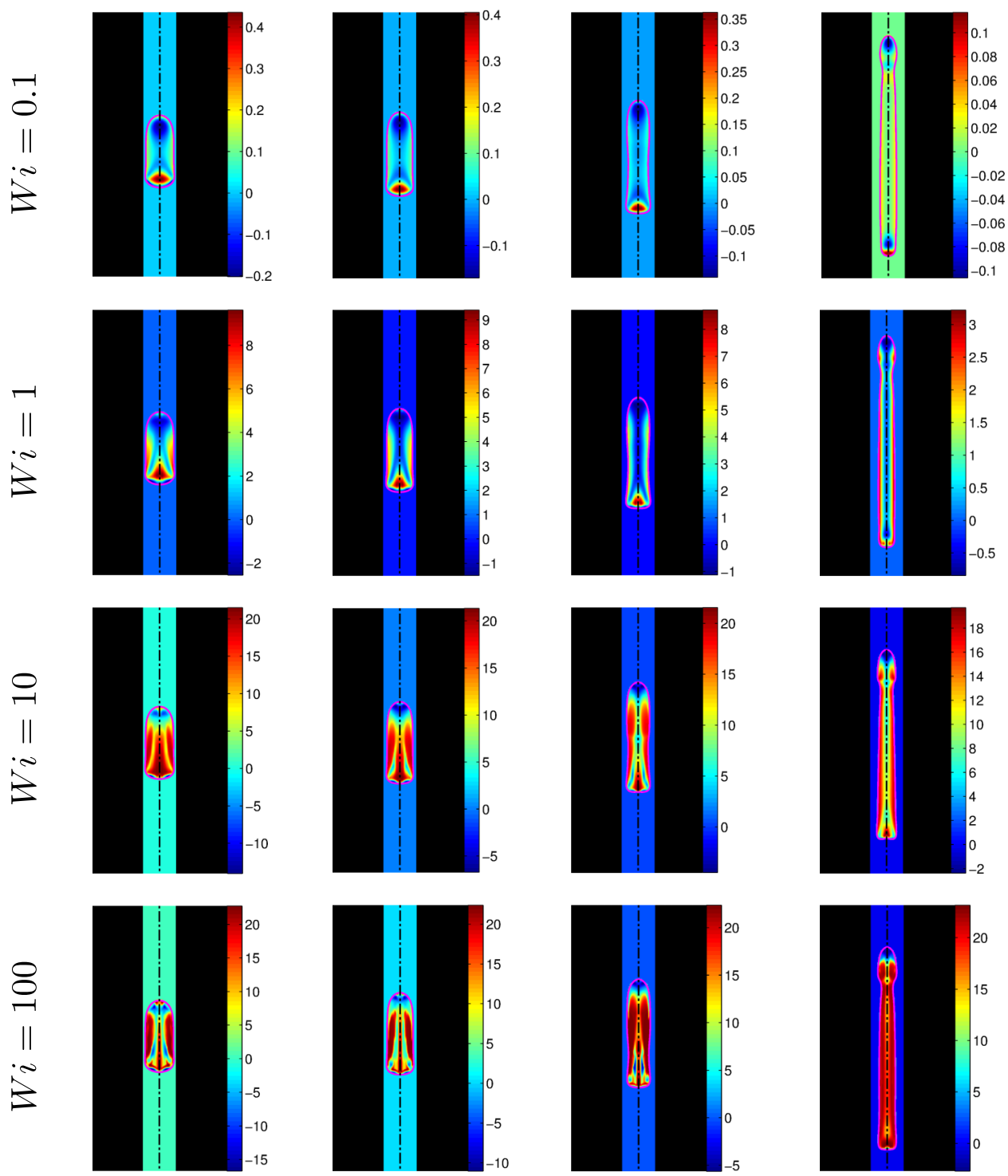

$$
C a=0.05
$$

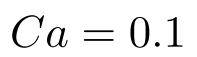

$$
C a=0.2
$$$$
C a=0.5
$$

FIG. 4. The effects of the capillary and Weissenberg numbers for the VN case. The droplet shapes and the constant contours of the difference between the normal components of the conformation tensor $A_{z z}-A_{r r}$ in the vicinity of the droplet in the middle of the constriction $(R e=2, \kappa=1.25$, grid: $64 \times 768)$. 

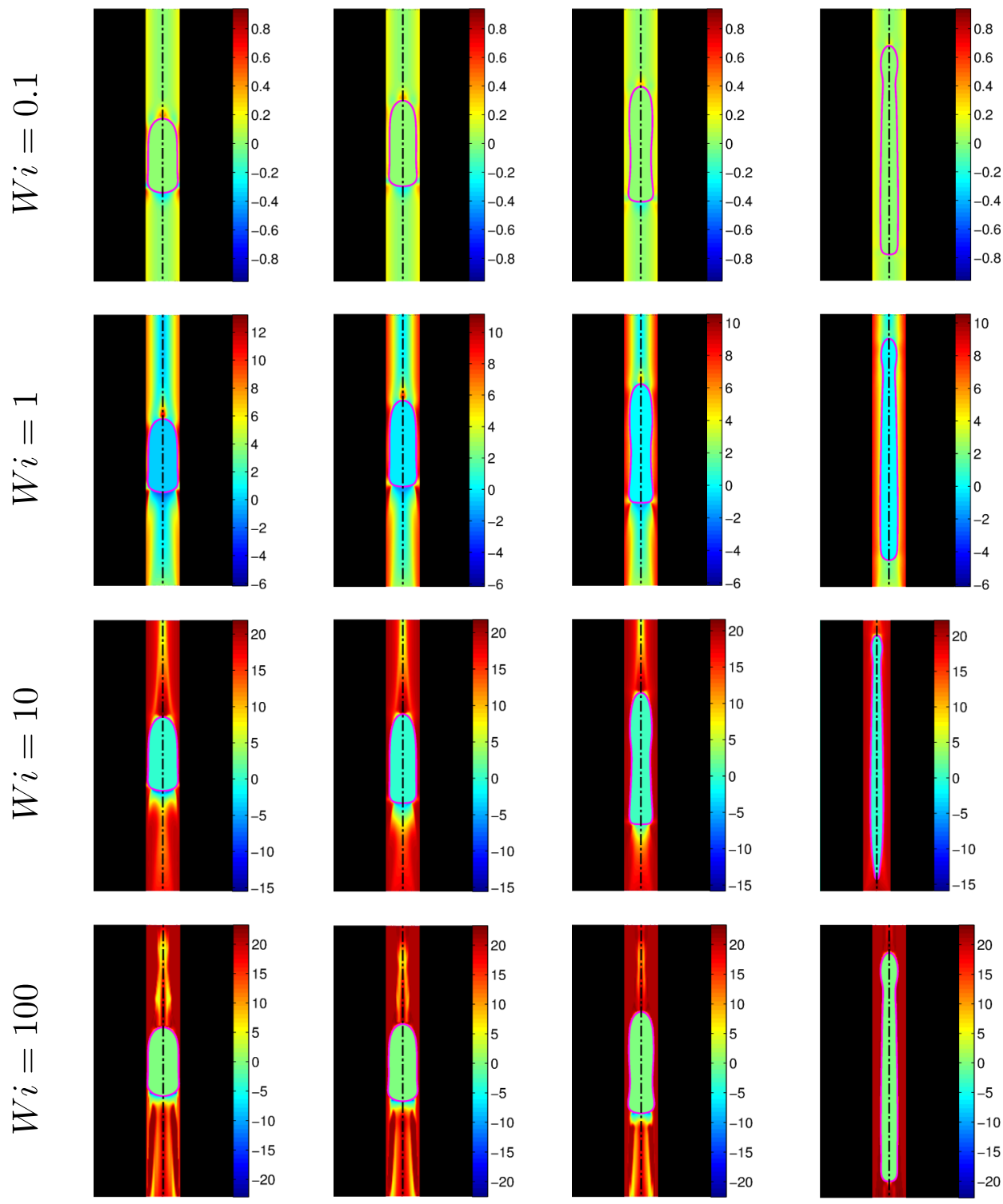

$C a=0.05$

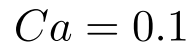

$C a=0.2$

$C a=0.5$

FIG. 5. The effects of the capillary and Weissenberg numbers for the NV case. The droplet shapes and the constant contours of the difference between the normal components of the conformation tensor $A_{z z}-A_{r r}$ in the vicinity of the droplet in the middle of the constriction. Note that $W i=10$ and $C a=0.5$ case is not in the same scale with the other snapshots $(R e=2$, $\kappa=1.25$, grid: $64 \times 768)$.

surface tension enhances drop deformation and reduces strain rate inside the droplet. Thus, for higher $\mathrm{Ca}$, the viscoelastic stresses inside the drop grow more slowly. This effect is more pronounced for $W i<10$. For up to a moderate $C a$ number (i.e., $0.05 \leq C a \leq 0.2$ ), drop deformation is affected far less by the viscoelasticity than the capillary forces. Viscoelastic stresses inside the drop at the rear stagnation point pull the interface inwards resulting in a slight indentation at the trailing edge. As $\mathrm{Ca}$ increases, the droplet becomes more elongated and its leading edge bulges. The bulge formation is caused by the surface tension that increases the pressure inside the high curvature at the tip of the interface and in turn pulls the tip towards the main body of droplet. ${ }^{35}$ As can be seen in Figs. 4 and 5, bulge formation occurs at $\mathrm{Ca}=0.5$. The bulge formation is more pronounced in the $\mathrm{VN}$ case since the viscoelastic stresses accumulate in the vicinity of neck region of the bulge (Fig. 4), which in turn enhances bulge formation by pulling the interface towards the front tip. 

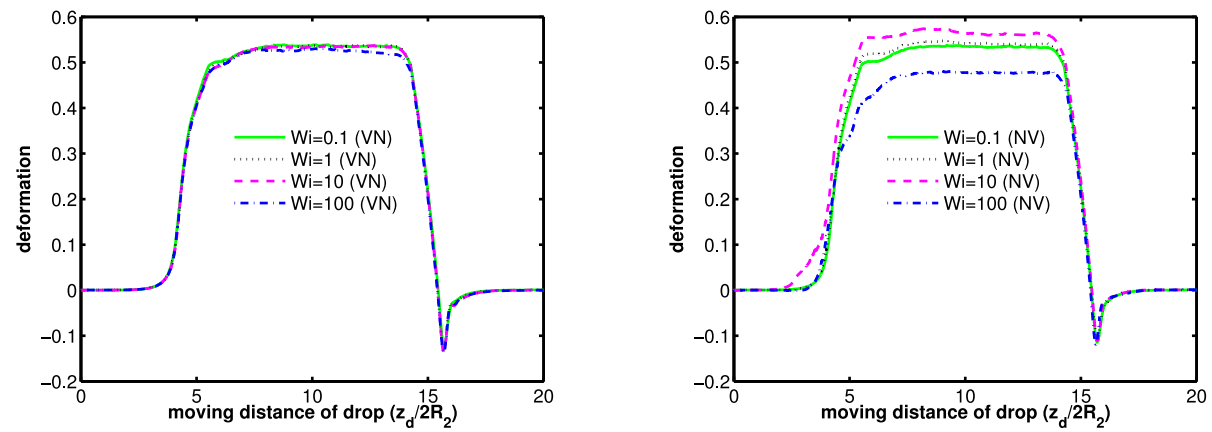

FIG. 6. Effects of fluid elasticity on drop deformation for the VN (left) and NV (right) cases. The deformation parameter is plotted against the moving distance of the drop centroid for various $W i$ ranging between $W i=0.1$ and $W i=100(C a=0.1$, $\operatorname{Re}=2, \kappa=1.25$, grid: $64 \times 768)$.

For the NV case, when the drop is in the constriction, large normal stress differences develop in the film region as well as the regions around rear and front stagnation points (Fig. 5). For low to moderate $\mathrm{Ca}(0.05 \leq \mathrm{Ca} \leq 0.1)$, the drop occupies major width of the constriction while, at high $\mathrm{Ca}$ $(\mathrm{Ca}=0.5)$, the drop forms a thin extended filament with a pointed end, occupying only a small portion of the constriction width. A notable difference with the $\mathrm{VN}$ case is that the trailing end of the drop is stretched out when $C a=0.5$ and $W i$ increases up to $W i=10$. This is mainly due to the stretching of the interface by polymer stresses concentrated around the rear stagnation point. For a higher $W i$ number $(W i>10)$, the viscoelastic stresses become stronger but penetrate over the entire cross section to make the stress distribution more uniform, which dramatically changes the drop deformation. The most striking effect is that the trailing end is not extended anymore at high Wi numbers even for $C a=0.5$. The non-monotonic droplet deformation has been also observed for a NV system in a shear flow by Yue et al. ${ }^{21}$ as well as Aggarwal and Sarkar, ${ }^{22}$ and in a converging pipe flow by Zhou et al. ${ }^{27}$ The deformation is plotted in Fig. 6 against the moving distance of the drop centroid for both the VN and NV systems. It is generally observed that the viscoelasticity has a weaker effect on drop deformation in the VN case than that in the NV case. In the NV case, the viscoelastic effects are non-monotonic, i.e., the deformation first increases and then decreases with Wi. Although not shown here, an increase in the elasticity ratio $k$ in the VV system decreases drop deformation resulting in a consistent pattern with that shown in Fig. 6.

\section{Effects of total viscosity ratio $(\theta)$}

We next examine the effects of drop-to-matrix viscosity ratio $\theta$. The droplet shapes and the constant contours of normal stress differences are plotted in Figs. 7 and 8 for the VN and NV cases,
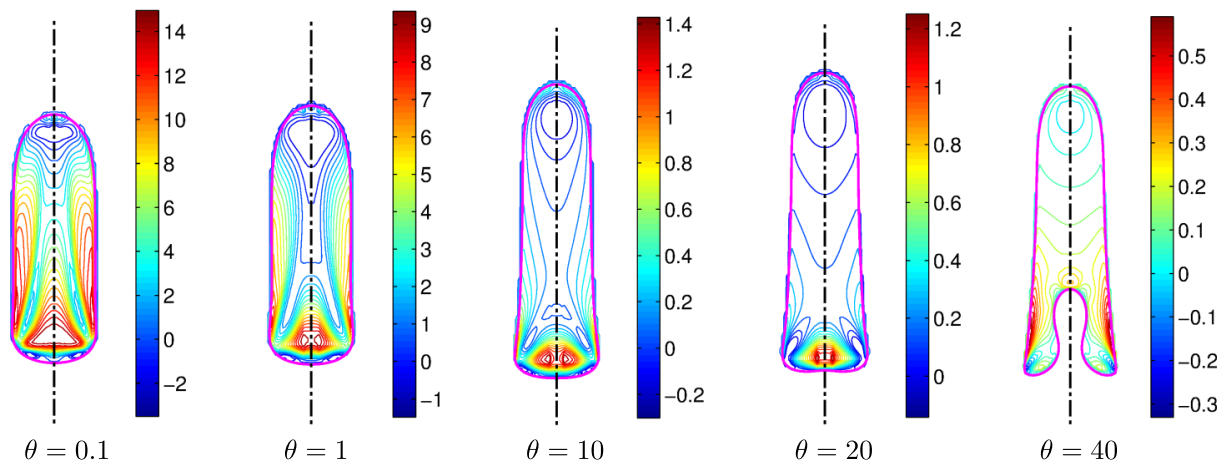

FIG. 7. The effects of viscosity ratio $(\theta)$ for the VN case. The droplet shapes and the constant contours of the difference between the normal components of the conformation tensor $A_{z z}-A_{r r}$ in the vicinity of the droplet in the constriction $(C a=0.1, R e=2, W i=1, \kappa=1.25$, grid: $64 \times 768)$. 


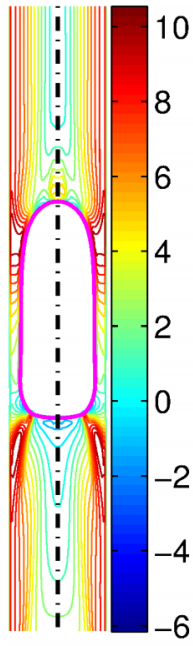

$\theta=0.1$

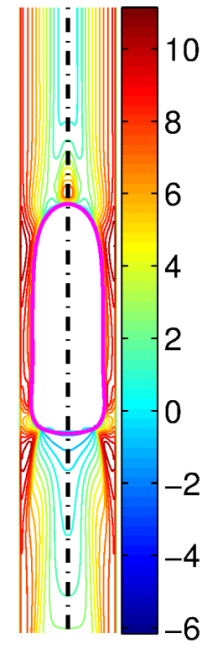

$\theta=1$

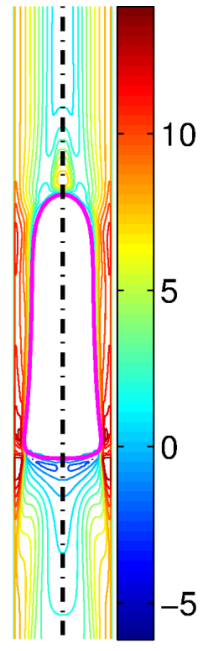

$\theta=10$

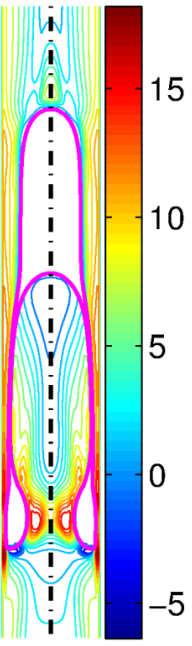

$\theta=20$

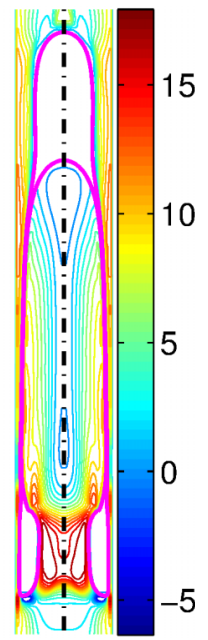

$\theta=40$

FIG. 8. The effects of viscosity ratio $(\theta)$ for the NV case. The droplet shapes and the constant contours of the difference between the normal components of the conformation tensor $A_{z z}-A_{r r}$ in the vicinity of the droplet in the constriction $(C a=0.1, R e=2, W i=1, \kappa=1.25$, grid: $64 \times 768)$.

respectively, in the middle of the constriction. These figures clearly show great sensitivity of drop deformation to $\theta$. As $\theta$ increases, the viscous normal stresses grow and eventually dominate over the combination of viscoelastic normal stresses and surface tension, leading to development of a re-entrant cavity. In a straight capillary tube, the viscoelasticity in drop/matrix fluid generally opposes/enhances drop deformation. However, as seen in Figs. 7 and 8, the re-entrant cavity occurs at a lower value of $\theta$ in the $\mathrm{NV}$ case compared to the VN system in the constricted channel. For instance, the re-entrant cavity forms in the NV system at $\theta=20$ (Fig. 8) while the drop remains intact for the VN system for the same viscosity ratio (Fig. 7). This is a direct result of the viscoelastic stress distribution at the back of the droplet when it enters the constriction as shown in Figs. 9 and 10 where the transient motion of a droplet is shown for the NV and VN cases, respectively. Note that the

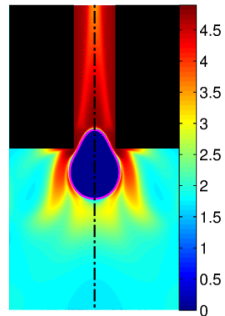

$t^{*}=54.7$

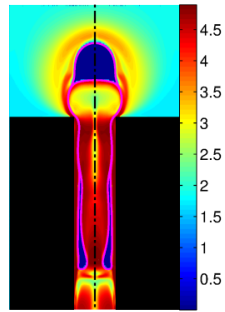

$t^{*}=74.8$

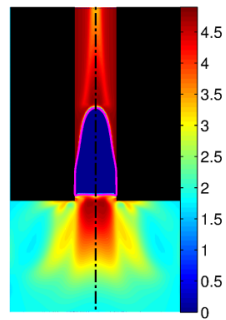

$t^{*}=57.5$

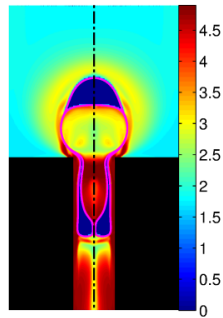

$t^{*}=77.5$

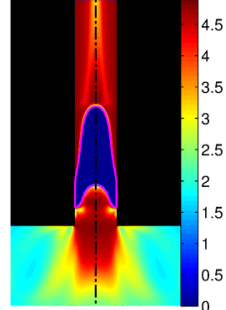

$t^{*}=58.4$

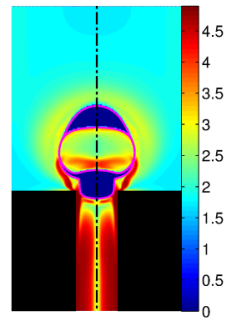

$t^{*}=80.3$

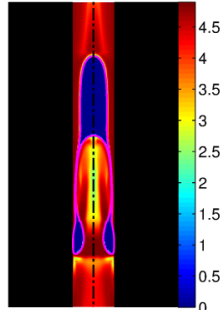

$t^{*}=64.8$

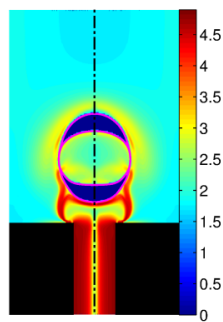

$t^{*}=83.0$

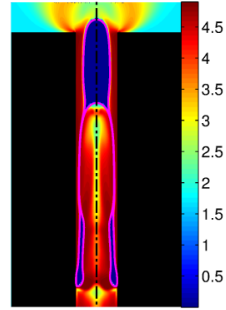

$t^{*}=69.3$

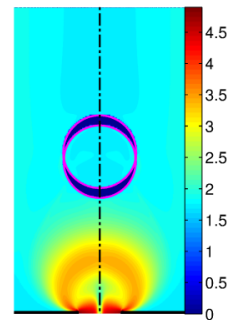

$t^{*}=119.5$

FIG. 9. A Newtonian droplet moving through a FENE-CR fluid in a pressure driven contraction/expansion capillary tube. The contours represent the average polymer extension $\sqrt{\operatorname{trace}(\mathbf{A})}(C a=0.1, R e=2, W i=10, \theta=20$, grid: $64 \times 768)$. 


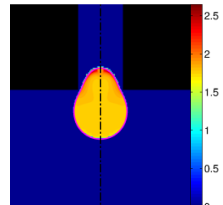

$t^{*}=65.7$

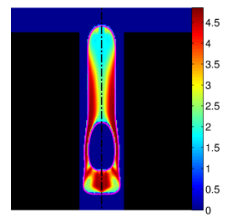

$t^{*}=80.3$

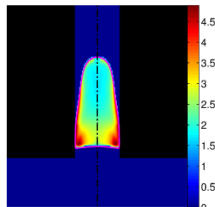

$t^{*}=68.4$

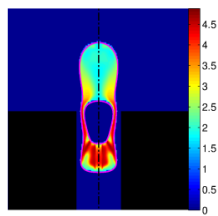

$t^{*}=83.9$

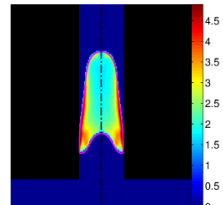

$t^{*}=69.3$

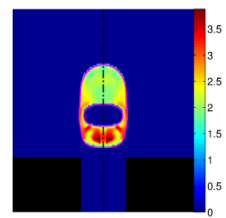

$t^{*}=86.6$

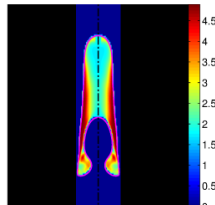

$t^{*}=73.9$

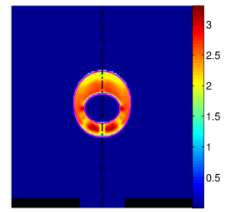

$t^{*}=95.8$

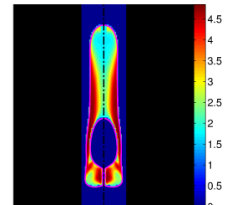

$t^{*}=78.4$

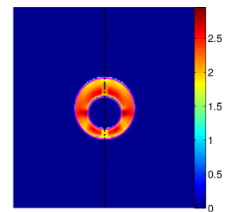

$t^{*}=123.1$

FIG. 10. A FENE-CR droplet moving through a Newtonian fluid in a pressure driven contraction/expansion capillary tube. The contours represent the average polymer extension $\sqrt{\operatorname{trace}(\mathbf{A})}(C a=0.1, R e=2, W i=100, \theta=40$, grid: 64 $\times 768)$.

Weissenberg number is increased in these figures to better demonstrate the stress distribution in the vicinity of the droplet. As seen, just before entering the constriction, a viscoelastic stress concentration occurs at the back of the droplet pushing the trailing edge to cause an indentation and subsequently a re-entrant cavity formation in the NV case. On the other hand, in the VN case, the viscoelastic stress concentration occurs at the shoulder of the droplet that acts against the viscous stresses to restore the indentation of the trailing edge. The re-entrant cavity grows continuously while the droplet is in the narrow channel and the entrained ambient fluid is eventually encapsulated within the main drop to form a bigger compound drop. The re-entrant cavity formation has been previously observed for all Newtonian ${ }^{53-55}$ and viscoelastic ${ }^{15}$ systems in circular tubes ${ }^{54,55}$ as well as in planar channels. ${ }^{15,53}$ Note that Harvie et al. ${ }^{15}$ reported only a few cases and only for a VN system in a similar constricted but a planar configuration. Once the indentation occurs at the entrance, the overall features of the re-entrant cavity formation and encapsulation are similar for both the VN and NV systems. The dorsal filaments created at the rear edge of the droplet excessively elongate especially for the NV system, which may even lead to a breakup. Note that the breakup is allowed only along the channel centerline in the present implementation of the numerical method and thus long posterior threads are not allowed to rupture. The dorsal filaments of the drop are thicker and shorter in the VN case than that in the NV case, so a posterior thread rupture is more likely to occur in the NV system. It is emphasized that, in spite of its great importance in applications, the drop breakup is not studied in details here since it is out of the scope of the present study.

It is evident that viscoelastic effects are important for the development of a puckered end and a re-entrant cavity. Therefore, further simulations are performed for the $\mathrm{VN}$ case for various $\mathrm{Wi}$ numbers, and the results are shown in Fig. 11. The viscosity ratio is fixed at $\theta=40$. It is interesting to observe that the viscoelasticity has a non-monotonic effect on the drop deformation, i.e., the size of the re-entrant cavity and resulting inner droplet first decreases and then increases with $W i$. This behavior is mainly attributed to the fact that the polymer stresses take a finite time $\left(T_{\text {elas }} \sim \lambda\right)$ to react to the flow. As mentioned before, the re-entrant cavity is essentially created in the entrance region. Since the flow time scale is proportional to the time required for the droplet to fully enter into the narrow channel, the relevant flow time scale is $T_{\text {flow }} \sim 2 R_{2} / V$. Thus, the flow time scale is much smaller than the viscoelastic response time, i.e., $T_{\text {flow }} \ll T_{\text {elas }}$ when $W i \gg 1$. As a result, the viscoelastic droplet behaves more like a Newtonian droplet in the entrance region for a sufficiently large Wi number.

The effects of viscosity ratio on drop deformation are quantified in Fig. 12 both for the VN and $\mathrm{NV}$ cases. The drop deformation increases with the viscosity ratio in both systems with a similar trend but a drop undergoes larger deformation in the NV system than that in the VN system. In the constriction region, a less viscous drop reaches a steady state very quickly while a more viscous drop continuously elongates and exits the constriction without reaching a steady motion. As demonstrated by Hooper et al. ${ }^{56}$ a more viscous drop reacts the exterior flow more slowly so its initial deformation lags that of a less viscous drop. However, it eventually attains larger deformation provided that 

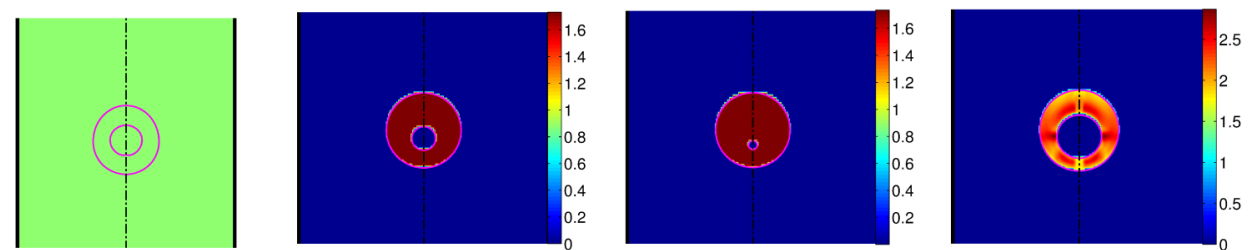

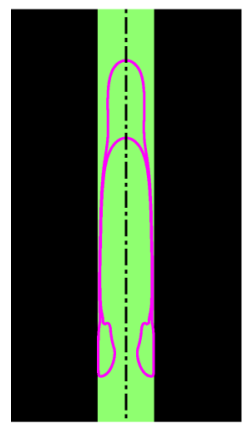

$W i=0$

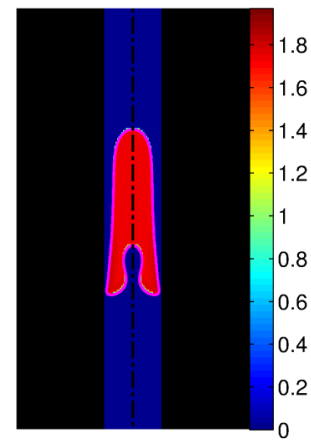

$W i=1$

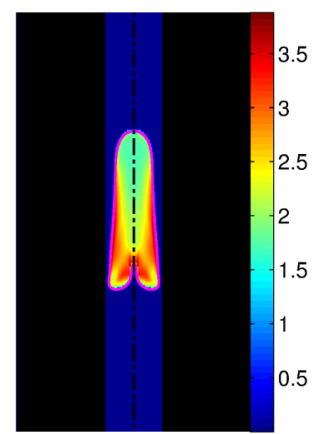

$W i=10$

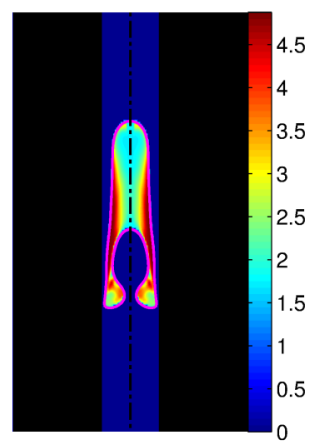

$W i=100$

FIG. 11. The effects of fluid elasticity on drop deformation at a high viscosity ratio of $\theta=40$ for the NN and VN cases. The droplet shapes and the constant contours of the average polymer extension $\sqrt{\operatorname{trace}(\mathbf{A})}$ are plotted in the vicinity of the droplet in the middle of the constriction (bottom plots) and in the further downstream of the expansion region (top plots) $(\mathrm{Ca}=0.1$, $R e=2, \kappa=1.25$, grid: $64 \times 768)$.

the capillary number is below the critical value for a breakup and the narrow section is sufficiently long. The slower initial reaction is due to a longer emulsion time (i.e., $\left.T_{\text {emulsion }}=\mu_{i} d_{d} / \sigma\right)^{57}$ and the greater steady-state deformation is due to the fact that more viscous interior sustains higher internal stresses. ${ }^{27}$ Similarly, the experimental study of Olbricht and Kung ${ }^{54}$ showed that the time scale for reaching a steady drop deformation increases with $\theta$. We finally note that the computational time is highly increased with $\theta$ due to time step restriction as well as the finer grid required to resolve very large drop deformations.

\section{Effects of extensibility $\left(L^{2}\right)$ and solvent viscosity ratio $(\beta)$}

Further simulations are carried out to examine the effects of solvent viscosity ratio and the extensibility parameter in the range of $0.1 \leq \beta \leq 0.9$ and $25 \leq L^{2} \leq 400$, respectively. Although not shown here due to space considerations, it is found that the effects of $\beta$ and $L^{2}$ are negligible for the VN case, so results are presented only for the NV system. The drop deformation is plotted in Fig. 13 for $W i=10$ and $\beta=0.1,0.5$, and 0.9 while keeping the other parameters the same as the base case.
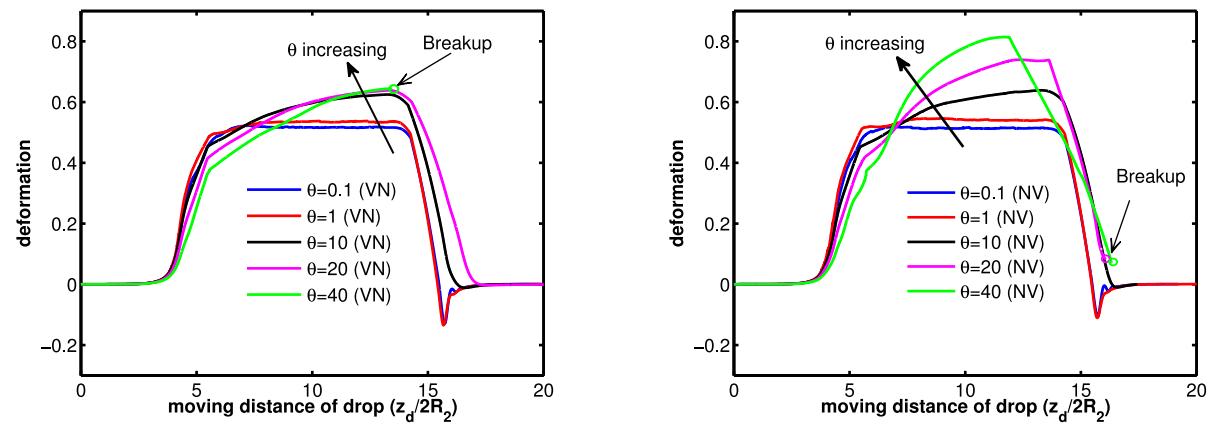

FIG. 12. The effects of viscosity ratio $(\theta)$ on drop deformation for the VN and NV cases. The deformation parameter is plotted against the moving distance of the drop centroid for various $\theta$ ranging between $\theta=0.1$ and $\theta=40(C a=0.1, R e=2$, $W i=1, \kappa=1.25)$. 

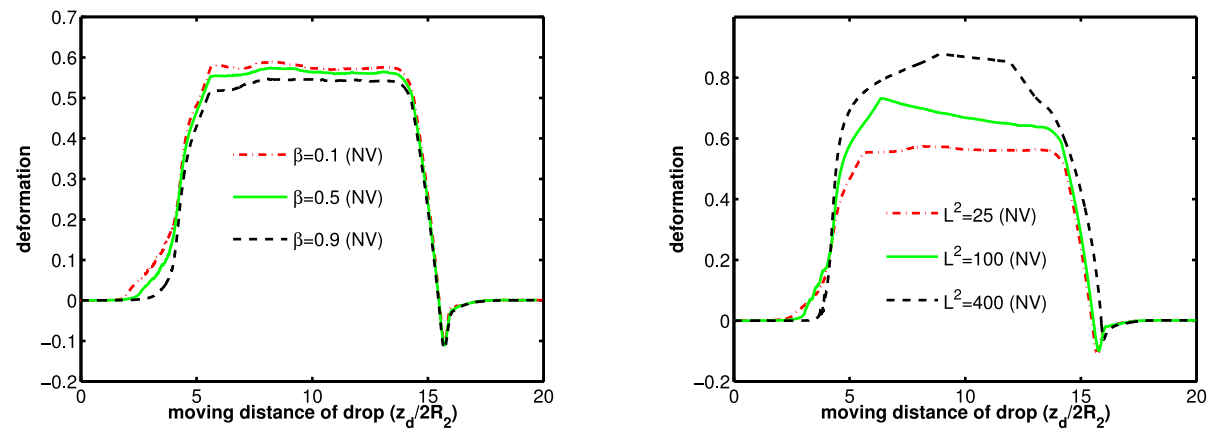

FIG. 13. The effects of solvent viscosity ratio $(\beta)$ (left plot) and extensibility $\left(L^{2}\right)$ (right plot) on drop deformation for the NV case. The deformation parameter is plotted against the moving distance of the drop centroid for various $\beta$ ranging between $\beta=0.1$ and $\beta=0.9$ and $L^{2}$ ranging between $L^{2}=25$ and $L^{2}=400(C a=0.1, R e=2, W i=10, \kappa=1.25$, grid: $64 \times 768)$.

In fact, the solvent viscosity ratio essentially modifies the effective Weissenberg number defined as $W i^{\prime}=W i(1-\beta)$, see, for instance, the work of Aggarwal and Sarkar. ${ }^{22}$ Thus, increasing $\beta$ has a similar effect as decreasing the Weissenberg number. The drop deformation generally increases as $\beta$ decreases, and the effects of $\beta$ are moderate for $W i=10$. Note that, although not shown here, $\beta$ has negligible influence on drop deformation for $W i=1$. Since the effective Weissenberg number increases with decreasing $\beta$ and the drop deformation is not very sensitive to $W i$ around $W i=1$, these results are consistent with the effects of $W i$ number (see, e.g., Fig. 6). Similar results are also found for the effects of $L^{2}$ on drop deformation as shown in Figs. 13 and 14. The larger the $L^{2}$, the more the molecule can be extended leading to enhanced viscoelastic effects. Viscoelastic stresses significantly increase with $L^{2}$ which in turn increase the deformation of the drop as shown in Figs. 13 and 14. A particular case is an infinite extension $\left(L^{2} \rightarrow \infty\right)$ which corresponds to the well-known Oldroyd-B model. These results are consistent with the fact that the viscoelasticity in the continuous phase enhances drop deformation as discussed before.

\section{B. Computations at high Reynolds numbers}

Finally, simulations are performed to study the role of viscoelasticity at high $R e$ for the VN, NV, and VV systems. Sample results are shown in Figs. 15 and 16 to show the overall droplet shape

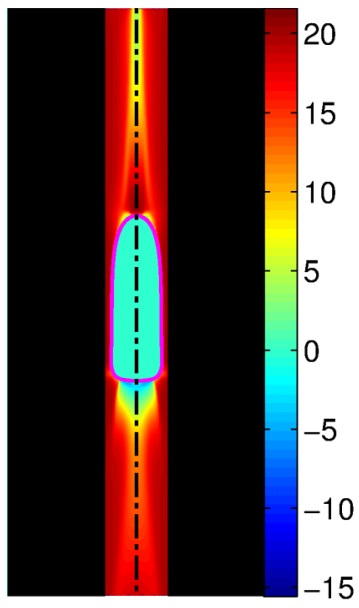

$L^{2}=25$

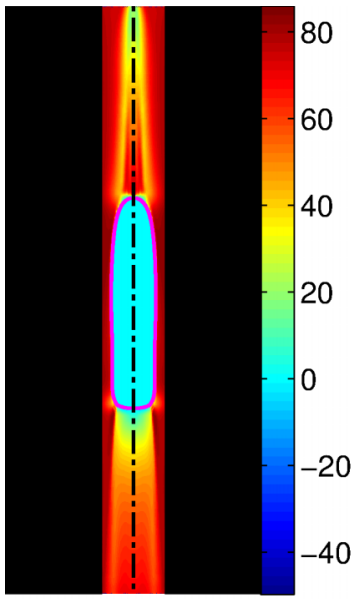

$L^{2}=100$

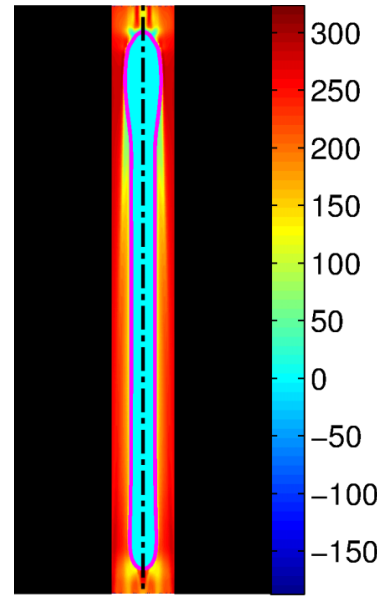

$L^{2}=400$

FIG. 14. The effects of extensibility $\left(L^{2}\right)$ on drop deformation for the NV case. The droplet shapes and the constant contours of the difference between the normal components of the conformation tensor $A_{z z}-A_{r r}$ are plotted in the vicinity of the droplet in the middle of the constriction ( $\mathrm{Ca}=0.1, \mathrm{Re}=2, \mathrm{Wi}=10, \kappa=1.25$, grid: $64 \times 768)$. 


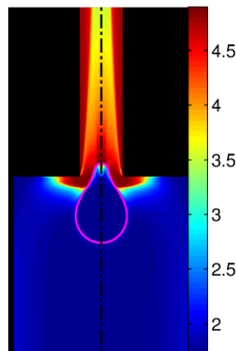

$t^{*}=87.6$

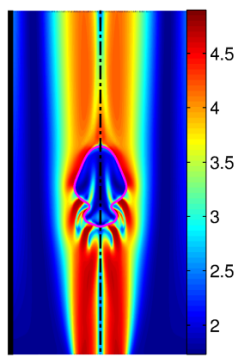

$t^{*}=112.2$

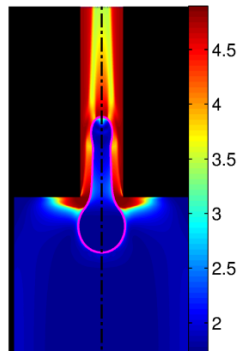

$t^{*}=90.3$

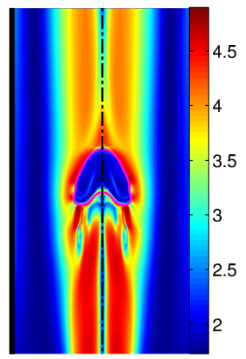

$t^{*}=114.9$

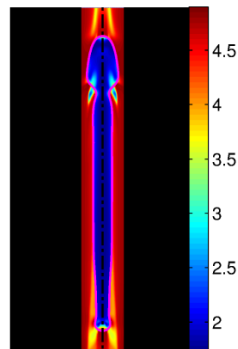

$t^{*}=99.4$

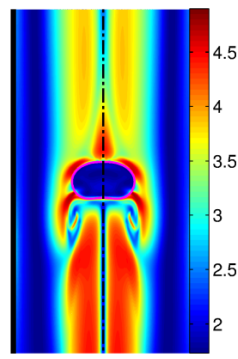

$t^{*}=122.2$

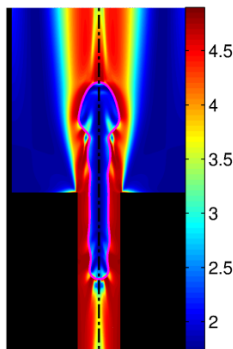

$t^{*}=105.8$

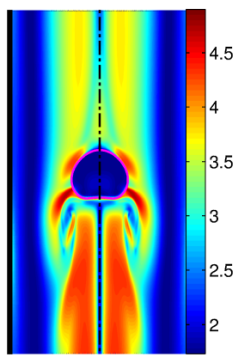

$t^{*}=126.8$

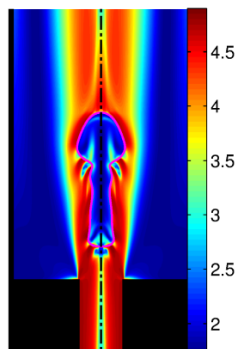

$t^{*}=108.5$

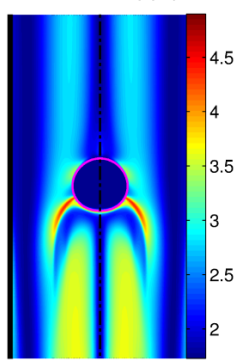

$t^{*}=172.4$

FIG. 15. A FENE-CR droplet moving through another FENE-CR fluid in a pressure driven contraction/expansion capillary tube at $W i_{i}=1$ and $W i_{o}=10$. The contours represent the average polymer extension $\sqrt{\operatorname{trace}(\mathbf{A})}(\mathrm{Ca}=0.1, R e=100$, grid: $96 \times 2496)$.

evolution together with the contours of average polymer extension for two different VV cases. The Reynolds number and fluid elasticity ratio are fixed at $R e=100$ and $k=0.1$ for both cases, while the inner and outer Weissenberg numbers are selected differently to demonstrate the effects of viscoelasticity, i.e., $\left(W i_{i}, W i_{o}\right)=(1,10)$ and $(10,100)$ in Figs. 15 and 16 , respectively. In both cases, as the droplet moves into the constriction, it first elongates and then retracts slowly. After entering the narrow channel, its leading edge bulges. The elongation is mainly due to the fact that the inertial

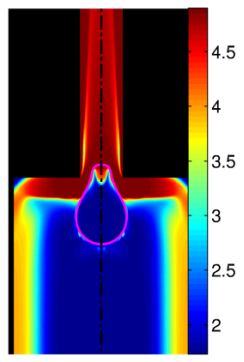

$t^{*}=90.3$

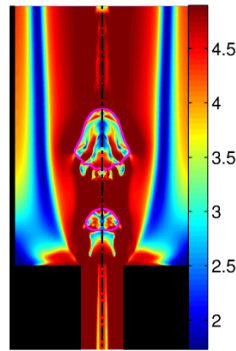

$t^{*}=112.2$

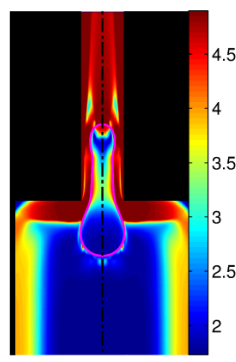

$t^{*}=93.0$

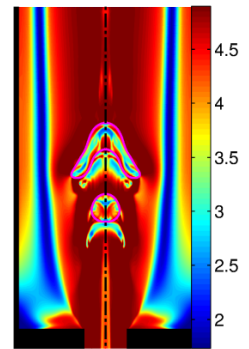

$t^{*}=114.9$

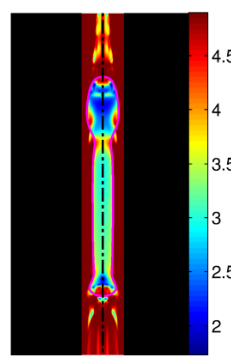

$t^{*}=102.2$

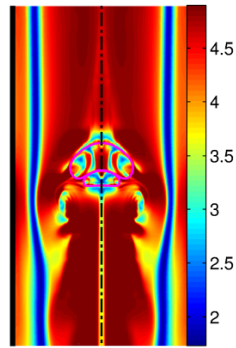

$t^{*}=125.9$

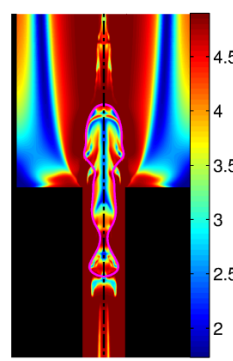

$t^{*}=108.5$

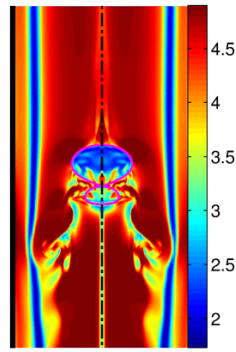

$t^{*}=135.0$

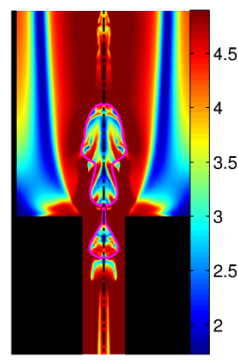

$t^{*}=109.9$

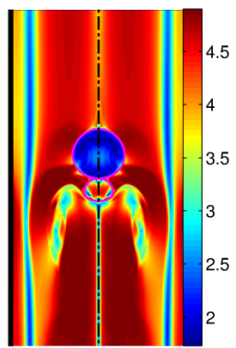

$t^{*}=166.9$

FIG. 16. A FENE-CR droplet moving through another FENE-CR fluid in a pressure driven contraction/expansion capillary tube at $W i_{i}=10$ and $W i_{o}=100$. The contours represent the average polymer extension $\sqrt{\operatorname{trace}(\mathbf{A})}(\mathrm{Ca}=0.1, \operatorname{Re}=100$, grid: $96 \times 2496$ ). 
forces become dominant over the other forces. The bulge formation occurs more severely at high $R e$ numbers as seen in Figs. 15 and 16. In addition, a bulge also forms at the trailing edge at high Wi numbers. At the trailing tip, the surface tension and viscoelastic stresses inside the droplet act in the same direction to increase the radius of the tip and thus to enhance the bulge formation. The stability of the bulge is determined by the competition between four forces: The viscous and the viscoelastic stresses in the ambient fluid are directed away from the bulk of the droplet promoting a breakup while viscoelastic stresses in the drop fluid and the surface tension act to pull the bulge back towards the bulk of the droplet and thus suppressing the breakup. Depending on the net resultant force, the bulge at the trailing edge may break up in some cases (Fig. 16).

Strong interactions between the inertia, surface tension, and viscoelastic stresses result in very rich and highly complicated dynamics both in the constriction and expansion regions. The droplet undergoes large deformation in the narrow channel followed by shape oscillations in the expansion region. These oscillations sustain for a long time in the downstream of the expansion, and thus require a long expansion pipe to reach a steady state. Similar oscillations were also observed by Carroll $^{43}$ for an all-Newtonian drop system in a capillary tube with a sudden expansion at high $R e$ numbers, i.e., up to $R e=200$. However, Carroll ${ }^{43}$ considered a relatively small droplet size (e.g., $\kappa=0.7$ ) so the magnitude of shape oscillations was significantly smaller than those in the present study. Drop breakup is also observed in the narrow channel near the expansion in some cases, see e.g., Fig. 16.

The effects of viscoelasticity on drop deformation at a high Reynolds number are further studied for the VN, NV, and VV systems. First simulations are carried out for the VN and NV cases at $R e=50$ and the Weissenberg number ranging between $W i=0$ (all Newtonian) and $W i=100$. Comparing Figs. 17 and 6 reveals that the inertia significantly enhances drop deformation especially in the constriction and expansion regions both for the VN and NV cases. Similar to the low $R e$ case, the effects of Wi on drop deformation are non-monotonic and more pronounced for the NV case in the narrow channel. However, unlike the low Re cases, drop deformation increases rapidly, reaches a maximum close to the entrance of the constriction, and then decreases continuously until it exits the narrow channel. The droplet undergoes large damped oscillations in the expansion region and reaches a steady motion after about $30 R_{2}$ in the downstream of the expansion. Wi has a non-monotonic effect on the shape oscillations in both the VN and NV cases. For the VN case, the amplitude of oscillation first increases and then decreases with Wi while it is just opposite for the NV case.

We next consider the VV case. For this purpose, first the Weissenberg number of the outer fluid is fixed at $W i_{o}=1$ and the simulations are performed for the Weissenberg numbers of the inner fluid $W i_{i}=0.1,1$, and 10 , which correspond to the elasticity ratio of $k=0.1,1$, and 10 , respectively. The Reynolds number is set to $R e=100$ and the results are shown in Fig. 18. The overall deformation pattern resembles the VN case for moderate Weissenberg numbers, see, e.g., Fig. 17. This is not surprising since the viscoelasticity of the outer fluid is kept fixed similar to the VN case with only
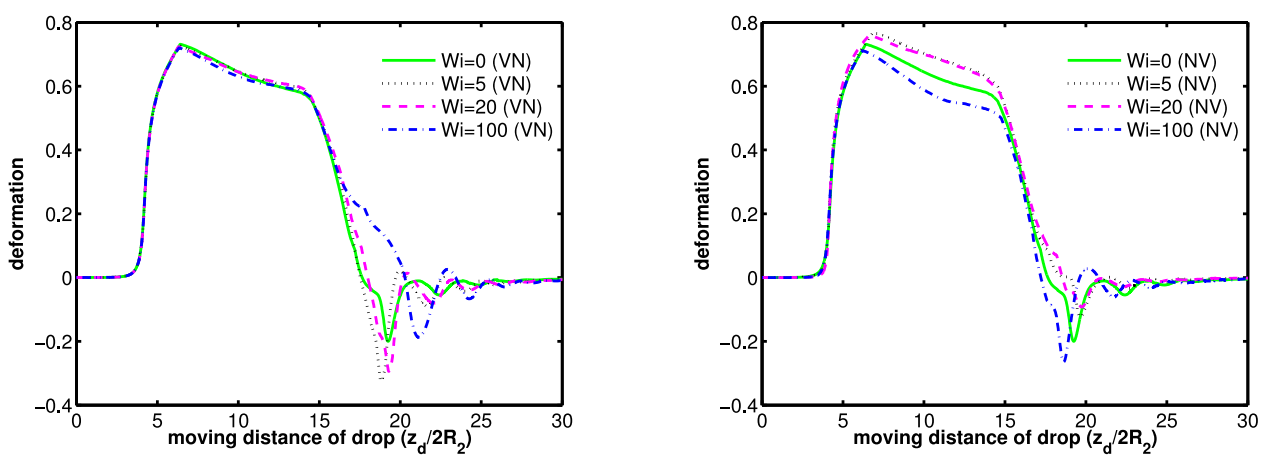

FIG. 17. The effects of fluid elasticity on drop deformation at a high Reynolds number $R e=50$ for the VN and NV cases. The deformation parameter is plotted against the moving distance of the drop centroid for various $W i$ numbers ranging between $W i=0$ (Newtonian) and $W i=100(C a=0.1, \kappa=1.25$, grid: $96 \times 2496)$. 

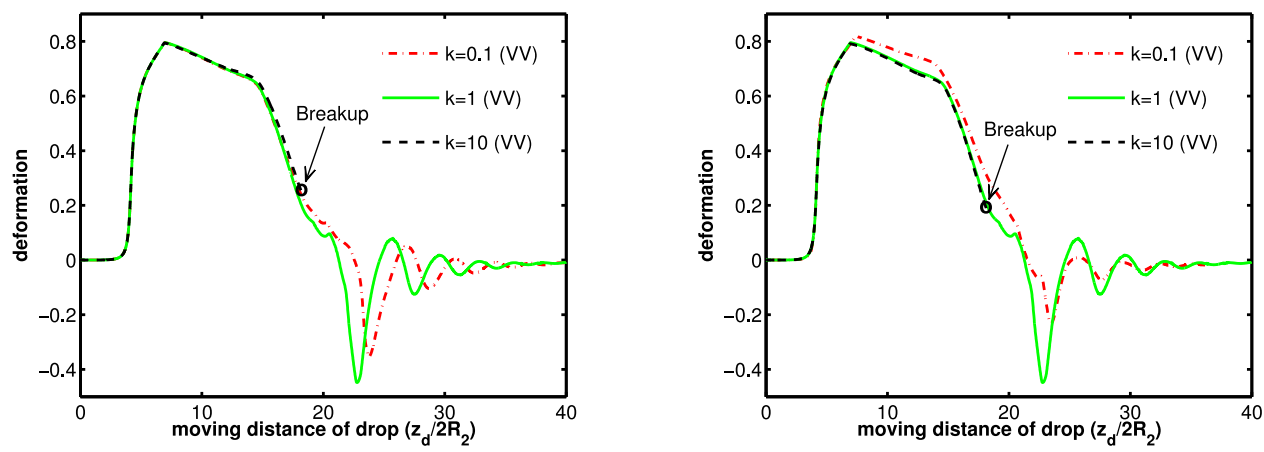

FIG. 18. Effects of fluid elasticity ratio $(k)$ on drop deformation. The inner and outer Weissenberg numbers are set to $W i_{o}=1$ and $W i_{i}=0.1,1,10$ (left) and $W i_{i}=1$ and $W i_{o}=0.1,1,10$ (right) for the VV cases. The deformation parameter is plotted against the moving distance of the drop centroid for various $k$ ranging between $k=0.1$ and $k=10(C a=0.1, R e=100$, $\kappa=1.25$, grid: $96 \times 2496)$.

difference being a non-zero value of the Weissenberg number. As $k$ increases, the amplitude of the oscillations in the expansion region increases. The drop deformation exceeds a critical value leading to a drop breakup in the constriction near the expansion region. Then simulations are also performed by fixing the inner Weissenberg number at $W i_{i}=1$ and varying the outer Weissenberg number as $W i_{o}=0.1,1$, and 10 (Fig. 18). This case resembles the NV system. The effects of $k$ on overall drop dynamics and deformation are similar to the previous case, i.e., the amplitude of surface oscillation in the expansion region increases as $k$ increases. Note that, for the same value of $k$, the drop undergoes a larger deformation for the case in which $W i_{o}$ is kept constant. Therefore, we can conclude that the values of $W i_{i}$ and $W i_{o}$ as well as their ratio $k$ are important for the VV case.

Finally, the sole effects of Reynolds number are examined both for the VN and NV cases. For this purpose, the Reynolds number is varied in the range $2 \leq R e \leq 100$ while fixing all other parameters at their values in the base case. The results are shown in Fig. 19 for the VN and NV cases. As can be seen in these figures, the drop deformation and amplitude of surface oscillations generally increase with $R e$. The surface oscillations exhibit a transition from an over damped regime for up to $R e=O(1)$ to an under damped regime for $R e>10$. Similar observations have also been made by Carroll ${ }^{43}$ for an all Newtonian case. In the expansion region, maximum amplitude of oscillation increases monotonically with a phase shift as $R e$ increases both for the $\mathrm{VN}$ and $\mathrm{NV}$ cases. Eventually a drop breakup occurs in the constriction for $R e=100$ and $W i=1$ in the VN case as indicated by a circle in Fig. 19. It can be seen that the trend is the same for all the systems but oscillations are more pronounced for the VN case.
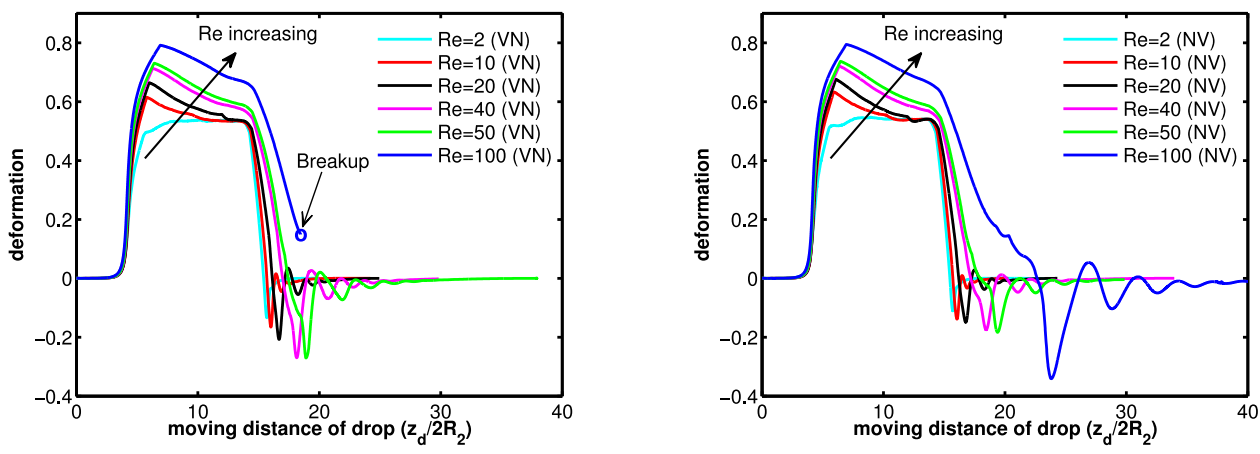

FIG. 19. Effects of inertia on drop deformation for the VN (left) and NV (right) cases. The deformation parameter is plotted against the moving distance of the drop centroid for various $R e$ ranging between $R e=2$ and $\operatorname{Re}=100(\mathrm{Ca}=0.1, \kappa=1.25$, Wi $=1$, grid: $96 \times 2496)$. 


\section{CONCLUSIONS}

The effects of viscoelasticity on drop dynamics and deformation in a pressure-driven capillary tube with a sudden contraction/expansion are studied computationally using a front-tracking method. A Newtonian drop in a viscoelastic medium (NV), a viscoelastic drop in a Newtonian medium (VN), and a viscoelastic drop in another viscoelastic medium (VV) cases are considered. The FENE-CR model is employed to account for viscoelasticity of inner and outer fluids. Extensive simulations are performed to examine the effects of relevant dimensionless parameters including the viscosity ratio $(\theta)$, the relative droplet size $(\kappa)$, the Reynolds number $(R e)$, and the capillary number $(C a)$ as well as the fluid elasticity characterized by the Weissenberg number $(W i)$, solvent viscosity ratio $(\beta)$, and the extensibility parameter $\left(L^{2}\right)$. In the VV case, the effects of the inner and outer Weissenberg numbers $\left(W i_{i}\right.$ and $\left.W i_{o}\right)$ as well as their ratio $(k)$ are also examined.

At a low Reynolds number $(R e=2)$, it is found that the drop deformation generally increases as the relative droplet size and capillary number increase similar to the all Newtonian system. The Weissenberg number is found to have a non-monotonic influence on drop dynamics for both the VN and NV systems. At a low to moderate Reynolds number $(R e<10)$, the drop deformation first increases/decreases as Wi increases up to $W i=O(10)$ and decreases/increases as $W i$ increases further for the $\mathrm{NV} / \mathrm{VN}$ case. At small $W i$, the viscoelastic stresses are mainly generated near the interface at the side of the drop and convected towards the trailing edge by the external/internal flow for the $\mathrm{NV} / \mathrm{VN}$ cases, leading to a stress concentration in the vicinity of the rear stagnation point. This stress concentration pulls out/in the drop interface to cause a pointed/rounded trailing edge in the NV/VN case. As $W i$ is further increased, e.g., $W i=100$, the viscoelastic stresses become more uniform in the dispersed/continuous phase and thus reduce the overall viscoelastic effects on drop deformation in the VN/NV system. We found that $L^{2}$ and $1-\beta$ have a similar effect and both increase drop deformation for the NV case. The effects of these parameters are found not to be very significant for the VN case. It is found that the viscosity ratio has a significant influence and generally increases drop deformation. A re-entrant cavity may develop at the back of the droplet as the viscosity ratio increases. In contrast with the straight channel case, the viscoelasticity in the drop phase hinders whereas in the continuous phase enhances the formation of a re-entrant cavity in the constricted channel. It is found that this unexpected behavior is primarily due to the viscoelastic stress distribution at the back of the droplet when the droplet enters the constriction. However, at sufficiently high $W i$, the droplet enters the narrow channel before the viscoelastic stresses are fully developed, so the droplet behaves more like a Newtonian droplet in the entrance region. The viscoelastic stresses then develop in the narrow channel and the viscoelastic effects resemble those in the two-phase viscoelastic systems in a straight tube.

At high $R e$, the droplet exhibits very rich dynamics with highly complicated deformation and stress distribution patterns. In particular, the droplet undergoes large deformation in the constriction followed by damped surface oscillations in the expansion region. Similar to the low Re case, Wi also has a non-monotonic effect on drop deformation at high $R e$ numbers. In the expansion region, the magnitude of surface oscillations first increases/decreases and then decreases/increases with $W i$ for the VN/NV case. The large deformation may even lead to a drop breakup in some cases, especially at high $R e$ and $W i$ numbers. The drop breakup occurs near the end of the narrow channel. The surface oscillations are generally more pronounced for the VN case. In the VV case, the surface oscillations generally increase with the elasticity ratio $(k)$ but the values of the inner and outer Weissenberg numbers are also found to be important for drop dynamics.

\section{ACKNOWLEDGMENTS}

The authors are grateful to the Scientific and Technical Research Council of Turkey (TUBITAK) for the support of this research through Grant No. 112M181 and Turkish Academy of Sciences (TUBA). The authors are grateful for the use of the computing facilities at TUBITAK-ULAKBIM, High Performance and Grid Computing Center. 


\section{APPENDIX: GRID CONVERGENCE}

Extensive computational simulations are performed to determine the minimum grid size required to reduce the spatial discretization error below a threshold value. Sample results are presented here for the low and high Reynolds numbers flows. For this purpose, first simulations are performed at low $R e$ for the VN and NV systems shown in Fig. 20 using various grid resolutions ranging between $32 \times 384$ and $128 \times 1536$. The evolution of drop deformation is plotted in Fig. 20. As can be seen in this figure, differences between successive grid resolutions decrease as grid is refined indicating grid convergence. The relative spatial error is also plotted in Fig. 20 at selected locations. For the grid size of $\Delta x$, the relative error is defined as

$$
\text { Error }=\left|\frac{\text { deformation }_{\Delta x \rightarrow 0}-\text { deformation }_{\Delta x}}{\text { deformation }_{\Delta x \rightarrow 0}}\right|,
$$

where deformation ${ }_{\Delta x}$ is the computed value and deformation $\operatorname{sx\rightarrow 0}_{\Delta x}$ is the spatial error free value predicted using the Richardson's extrapolation. Note that, although not shown here, the drop volume is preserved within $2 \%$ for all the cases considered in this study. The approximate linear relationship confirms the expected second order spatial accuracy of the method. These figures show that a $64 \times 768$ grid is required to reduce the maximum spatial error below $2 \%$ in the drop deformation. Note that the results for low $R e$ presented in this paper are obtained using a $64 \times 768$ grid for which the maximum spatial error is reduced below $4 \%$ in all the flow quantities of interest. Finally, the mesh convergence analysis is also done for high $R e$ flows both for $\mathrm{VN}$ and $\mathrm{NV}$ cases using various grid resolutions ranging between $32 \times 832$ and $128 \times 3328$. The results are shown in Fig. 21. It is found that a grid convergence is achieved with expected second order accuracy and a $96 \times 2496$ grid is sufficient to reduce the spatial error below $2 \%$ in all the flow quantities except for the VN system at
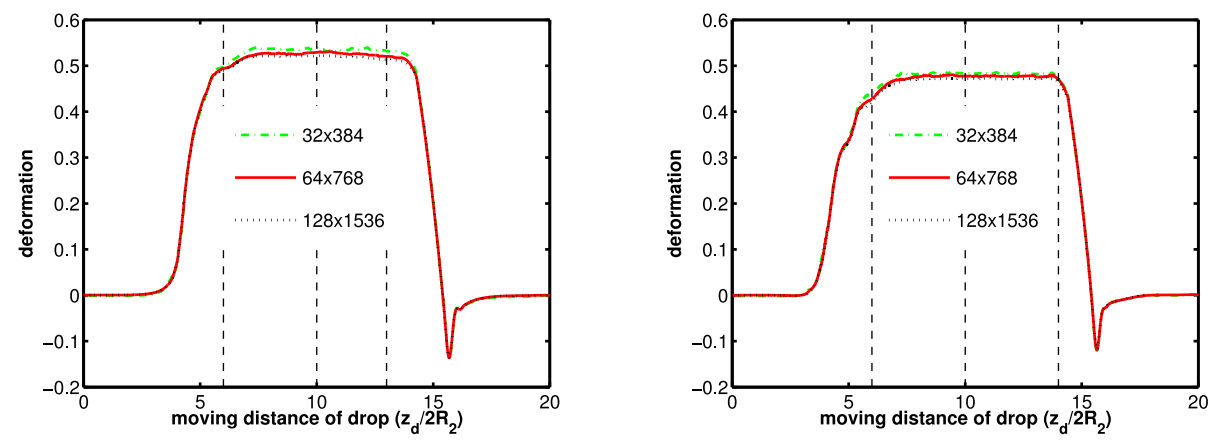

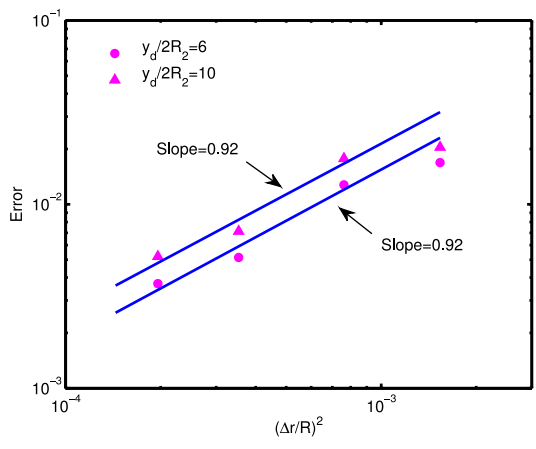

(a) VN Case

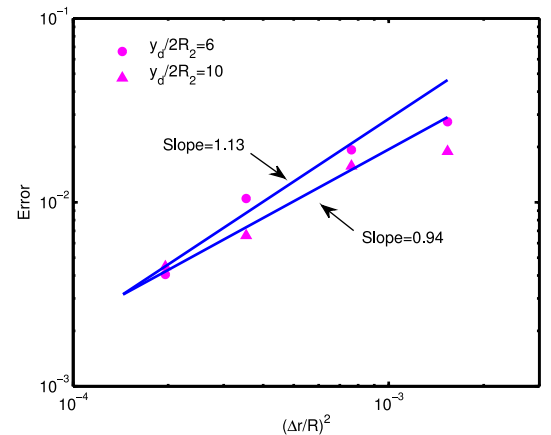

(b) NV Case

FIG. 20. Grid convergence for the $\mathrm{VN}$ and $\mathrm{NV}$ cases at $R e=2$. The results are obtained using various grid resolutions ranging between $32 \times 384$ and $128 \times 1536$. The drop deformation versus the moving distance of the drop centroid (top row) and the variation of error in drop deformation with the square of the non-dimensional grid size $(\Delta r / R)^{2}$ at the axial locations $y_{d} / 2 R_{2}=6$ and 10 (bottom row) for (a) the VN and (b) the NV cases, respectively. The solid lines are the linear least squares fits to the computational results indicating the expected second-order accuracy of the method $(\mathrm{Ca}=0.1, \mathrm{Wi}=100)$. 

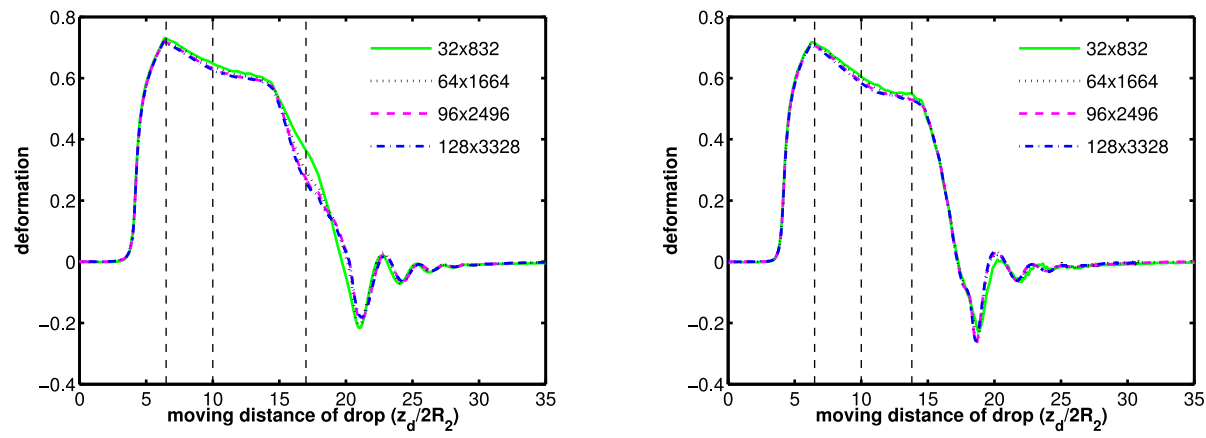

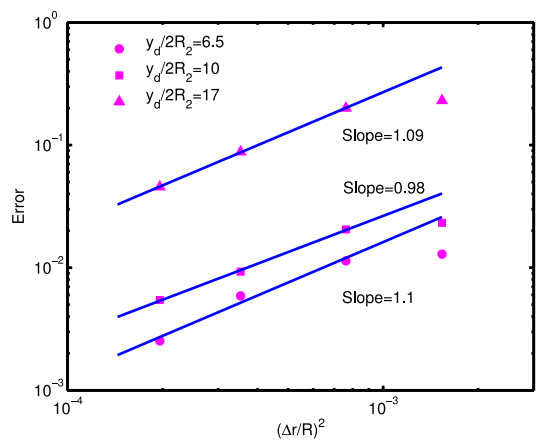

(a) VN Case

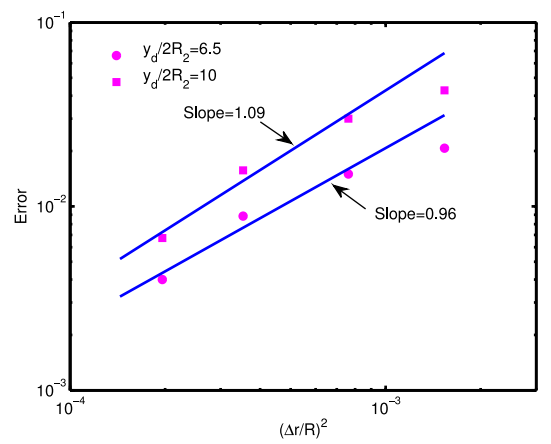

(b) NV Case

FIG. 21. Grid convergence for the VN and NV cases at $R e=50$. The results are obtained using various grid resolutions ranging between $32 \times 832$ and $128 \times 3328$. The drop deformation versus the moving distance of the drop centroid (top row) and the variation of error in drop deformation with the square of the non-dimensional grid size $(\Delta r / R)^{2}$ at the axial locations $y_{d} / 2 R_{2}=6.5,10,17$ and $y_{d} / 2 R_{2}=6.5,10$ (bottom row) for (a) the VN and (b) the NV cases, respectively. The solid lines are the linear least squares fits to the computational results indicating the expected second-order accuracy of the method $(C a=0.1, W i=100)$.

high $W i$ numbers, i.e., in the extreme case of $W i=100$, the relative error becomes as large as $9 \%$ in the vicinity of the expansion.

${ }^{1}$ T. M. Squires and S. R. Quake, "Microfluidics: Fluid physics at the nanoliter scale," Rev. Mod. Phys. 77(3), 977 (2005).

${ }^{2}$ H. A. Stone, A. D. Stroock, and A. Ajdari, "Engineering flows in small devices: Microfluidics toward a lab-on-a-chip," Annu. Rev. Fluid Mech. 36, 381 (2004).

${ }^{3}$ W. L. Olbricht, "Pore-scale prototypes of multiphase flow in porous media," Annu. Rev. Fluid Mech. 28, 128 (1996).

${ }^{4}$ A. M. Forsyth, J. Wan, W. D. Ristenpart, and H. A. Stone, "The dynamic behavior of chemically stiffened red blood cells in microchannel flows," Microvasc. Res. 80, 37 (2010).

${ }^{5}$ S. M. Hosseini and J. J. Feng, "A particle-based model for the transport of erythrocytes in capillaries," Chem. Eng. Sci. 64, 4488 (2009).

${ }^{6}$ T. Wu and J. J. Feng, "Simulation of malaria-infected red blood cells in microfluidic channels: Passage and blockage," Biomicrofluidics 7, 044115 (2013).

${ }^{7}$ Z. Zhang, J. Xi, B. Hong, and X. Chen, "The effects of 3D channel geometry on CTC passing pressure-towards deformabilitybased cancer cell separation," Lab Chip 14, 2576 (2014).

${ }^{8}$ C. D. Han, Multiphase Flow in Polymer Processing (Academic Press, New York, 1981).

${ }^{9}$ A. Groisman, M. Enzelberger, and S. R. Quake, "Microfluidic memory and control devices," Science 300, 955 (2003).

${ }^{10}$ A. Groisman and S. R. Quake, "A microfluidic rectifier: Anisotropic flow resistance at low Reynolds numbers," Phys. Rev. Lett. 92, 094501 (2004).

${ }^{11}$ D. Dendukuri, K. Tsoi, T. A. Hatton, and P. S. Doyle, "Controlled synthesis of nonspherical microparticles using microfluidics," Langmuir 21, 2113 (2005).

12 A. Groisman and V. Steinberg, "Efficient mixing at low Reynolds numbers using polymer additives," Nature 410, 905 (2001).

${ }^{13}$ R. Cardinaels, S. Afkhami, Y. Renardy, and P. Moldenaers, "An experimental and numerical investigation of the dynamics of microconfined droplets in systems with one viscoelastic phase," J. Non-Newtonian Fluid Mech. 166, 52 (2011).

${ }^{14}$ L. Derzsi, M. Kasprzyk, J. P. Plog, and P. Garstecki, "Flow focusing with viscoelastic liquids," Phys. Fluids 25, 092001 (2013).

${ }^{15}$ D. J. E. Harvie, J. J. Cooper-White, and M. R. Davidson, "Deformation of a viscoelastic droplet passing through a microfluidic contraction,” J. Non-Newtonian Fluid Mech. 155, 67 (2008). 
${ }^{16}$ A. Gupta and M. Sbragaglia, "Deformation and breakup of viscoelastic droplets in confined shear flow," Phys. Rev. E 90, 023305 (2014).

17 J. J. Elmendorp and R. J. Maalcke, “A study on polymer blending microrheology: Part I," Polym. Eng. Sci. 25, 1041 (1985)

${ }^{18}$ R. W. Flumerfelt, "Drop breakup in simple shear fields of viscoelastic fluids," Ind. Eng. Chem. Fundam. 11, 312 (1972).

${ }^{19}$ F. Mighri, A. Ajji, and P. J. Carreau, "Influence of elastic properties on drop deformation in elongational flow," J. Rheol. 41, 1183 (1997).

${ }^{20}$ F. Mighri and P. J. Carreau, "Influence of elastic properties on drop deformation and breakup in shear flow," J. Rheol. 42, 1477 (1998).

${ }^{21}$ P. Yue, J. J. Feng, C. Liu, and J. Shen, “Viscoelastic effects on drop deformation in steady shear," J. Fluid Mech. 540, 427 (2005).

${ }^{22}$ N. Aggarwal and K. Sarkar, "Effects of matrix viscoelasticity on viscous and viscoelastic drop deformation in a shear flow," J. Fluid Mech. 601, 63 (2008).

${ }^{23}$ S. Ramaswamy and L. G. Leal, "The deformation of a Newtonian drop in the uniaxial extensional flow of a viscoelastic liquid," J. Non-Newtonian Fluid Mech. 88, 149 (1999).

${ }^{24}$ S. Ramaswamy and L. G. Leal, "The deformation of a viscoelastic drop subjected to steady uniaxial extensional flow of a Newtonian fluid," J. Non-Newtonian Fluid Mech. 85, 127 (1999).

${ }^{25}$ H. B. Chin and C. D. Han, "Studies on droplet deformation and breakup. I. Droplet deformation in extensional flow," J. Rheol. 23, 557 (1979).

${ }^{26}$ R. E. Khayat, "Boundary element analysis of planar drop deformation in confined flow. Part II. Viscoelastic fluids," Eng. Anal. Boundary Elem. 22, 291 (1998).

27 D. Zhou, P. Yue, and J. Feng, "Viscoelastic effects on drop deformation in a converging pipe flow," J. Rheol. 522, 469 (2008).

${ }^{28}$ C. Chung, M. Lee, K. Char, K. H. Ahn, and S. J. Lee, "Droplet dynamics passing through obstructions in confined microchannel flow," Microfluid. Nanofluid. 9, 1151 (2010).

${ }^{29}$ M. Lee, W. Park, C. Chung, J. Lim, S. Kwon, K. H. Ahn, S. J. Lee, and K. Char, "Multilayer deposition on patterned posts using alternating polyelectrolyte droplets in a microfluidic device," Lab Chip 10, 1160 (2010).

${ }^{30}$ B. P. Ho and L. G. Leal, "The creeping motion of liquid drops through a circular tube of comparable diameter," J. Fluid Mech. 71, 361 (1975).

${ }^{31}$ S. S. Khobdeh, Ph.D. thesis, Pennsylvania State University, 2011.

${ }^{32}$ W. L. Olbricht and L. G. Leal, "The creeping motion of liquid drops through a circular tube of comparable diameter: The effect of density differences between the fluids," J. Fluid Mech. 115, 187 (1982).

${ }^{33}$ H. Wu, Ph.D. thesis, University of Virginia, 2008.

${ }^{34}$ R. You, H. Haj-Hariri, and A. Borhan, "Confined drop motion in viscoelastic two-phase systems," Phys. Fluids 21, 013102 (2009).

${ }^{35}$ D. J. E. Harvie, M. R. Davidson, J. J. Cooper-White, and M. Rudman, "A parametric study of droplet deformation through a microfluidic contraction," ANZIAM J. 46(E), C150 (2005).

${ }^{36}$ D. J. E. Harvie, M. R. Davidson, J. J. Cooper-White, and M. Rudman, "A parametric study of droplet deformation through a microfluidic contraction: Low viscosity Newtonian droplets," Chem. Eng. Sci. 61, 5149 (2006).

${ }^{37}$ D. J. E. Harvie, M. R. Davidson, J. J. Cooper-White, and M. Rudman, "A parametric study of droplet deformation through a microfluidic contraction: Shear thinning liquids," Int. J. Multiphase Flow 33, 545 (2007).

${ }^{38}$ C. Zhou, P. Yue, and J. J. Feng, "Simulation of neutrophil deformation and transport in capillaries using Newtonian and viscoelastic drop models," Ann. Biomed. Eng. 35, 766 (2007).

${ }^{39}$ C. Chung, M. A. Hulsen, J. M. Kim, K. H. Ahn, and S. J. Lee, "Numerical study on the effect of viscoelasticity on drop deformation in simple shear and 5:1:5 plannar contraction/expansion microchannel," J. Non-Newtonian Fluid Mech. 155, 80 (2008)

${ }^{40}$ C. Chung, J. M. Kim, M. A. Hulsen, K. H. Ahn, and S. J. Lee, "Effect of viscoelasticity on drop dynamics in 5:1:5 plannar contraction/expansion microchannel flow," Chem. Eng. Sci. 64, 4515 (2009).

${ }^{41}$ H. Amini, W. Lee, and D. Di Carlo, "Inertial microfluidic physics,” Lab Chip 14, 2739 (2014).

42 D. Di Carlo, "Inertial microfluidics," Lab Chip 9, 3038 (2009).

${ }^{43}$ R. Carroll, Ph.D. thesis, University of New Hampshire, 2014.

${ }^{44}$ K. S. Sheth and C. Pozrikidis, "Effects of inertia on the deformation of liquid drops in simple shear flow," Comput. Fluids 24, 101 (1995).

${ }^{45}$ D. Izbassarov and M. Muradoglu, "A front-tracking method for computational modeling of viscoelastic two-phase systems," J. Non-Newtonian Fluid Mech. 223, 122 (2015).

${ }^{46}$ S. O. Unverdi and G. Tryggvason, "A front-tracking method for viscous, incompressible, multi-fluid flows," J. Comput. Phys. 100, 25 (1992).

${ }^{47}$ M. D. Chilcott and J. M. Rallison, “Creeping flow of dilute polymer solutions past cylinders and spheres,” J. Non-Newtonian Fluid Mech. 29, 381 (1988).

48 A. R. Chorin, "Numerical solution of the Navier-Stokes equations," Math. Comput. 22, 745 (1968).

${ }^{49}$ R. Fattal and R. Kupferman, "Time-dependent simulation of viscoelastic flows at high Weissenberg number using the log-conformation representation,” J. Non-Newtonian Fluid Mech. 126, 23 (2005).

${ }^{50}$ R. Borges, M. Carmona, B. Costa, and W. S. Don, "An improved weighted essentially non-oscillatory sheme for hyperbolic conservation laws," J. Comput. Phys. 227, 3191 (2008).

${ }^{51}$ G. Tryggvason, B. Bunner, A. Esmaeeli, D. Juric, N. Al-Rawahi, W. Tauber et al., "A front-tracking method for the computations of multiphase flow," J. Comput. Phys. 169, 708 (2001).

${ }^{52}$ M. J. Martinez and K. S. Udell, "Axisymmetric creeping motion of drops through circular tubes," J. Fluid Mech. 210, 565 (1990).

${ }^{5}$ A. J. Griggs, A. Z. Zinchenko, and R. H. Davis, "Low-Reynolds-number motion of a deformable drop between two parallel plane walls,” Int. J. Multiphase Flow 33, 182 (2007). 
${ }^{54}$ W. L. Olbricht and D. M. Kung, "The deformation and breakup of liquid drops in low Reynolds number flow through a capillary," Phys. Fluids 4, 1347 (1992).

55 T. M. Tsai and M. J. Miksis, "Dynamics of a drop in a constricted capillary tube," J. Fluid Mech. 274, 197 (1994).

${ }^{56}$ R. W. Hooper, V. F. de Almeida, C. W. Macosko, and J. J. Derby, "Transient polymeric drop extension and retraction in uniaxial extensional flows," J. Non-Newtonian Fluid Mech. 98, 141 (2001).

${ }^{57}$ V. Sibillo, M. Simeone, and S. Guido, "Break-up of a Newtonian drop in a viscoelastic matrix under simple shear flow," Rheol. Acta 43, 449 (2004). 